\title{
An oscillation free shock-capturing method for compressible van der Waals supercritical fluid flows
}

\author{
C. Pantano ${ }^{\mathrm{a}, *}$, R. Saurel ${ }^{\mathrm{b}, \mathrm{c}}$, T. Schmitt ${ }^{\mathrm{d}, \mathrm{e}}$ \\ ${ }^{a}$ University of Illinois at Urbana-Champaign, Department of Mechanical Science and Engineering, 2115 Mechanical \\ Engineering Laboratory, 1206 W. Green St. Urbana, Il, 61801, USA \\ ${ }^{b}$ Aix Marseille University and University Institute of France, CNRS, Centrale Marseille, M2P2 UMR 7340, 38 rue Joliot \\ Curie, 13451 Marseille Cedex 13, France \\ ${ }^{c}$ RS2N SAS, 371 chemin de Gaumin, 83640 Saint Zacharie, France \\ ${ }^{d}$ Ecole Centrale Paris, Grande Voie des Vignes, 92295 Chatenay-Malabry, France \\ ${ }^{e}$ CNRS, UPR 288, Laboratoire d'Energétique Moléculaire et Macroscopique, Combustion (EM2C), Grande Voie des Vignes, \\ 92295 Chatenay-Malabry, France
}

\begin{abstract}
Numerical solutions of the Euler equations using real gas equations of state (EOS) often exhibit serious inaccuracies. The focus here is the van der Waals EOS and its variants (often used in supercritical fluid computations). The problems are not related to a lack of convexity of the EOS since the EOS are considered in their domain of convexity at any mesh point and at any time. The difficulties appear as soon as a density discontinuity is present with the rest of the fluid in mechanical equilibrium and typically result in spurious pressure and velocity oscillations. This is reminiscent of well-known pressure oscillations occurring with ideal gas mixtures when a mass fraction discontinuity is present, which can be interpreted as a discontinuity in the EOS parameters. We are concerned with pressure oscillations that appear just for a single fluid each time a density discontinuity is present. The combination of density in a nonlinear fashion in the EOS with diffusion by the numerical method results in violation of mechanical equilibrium conditions which are not easy to eliminate, even under grid refinement.

A cure to this problem is developed in the present paper for the van der Waals EOS based on previous ideas. A special extra field and its corresponding evolution equation is added to the flow model. This new field separates the evolution of the nonlinear part of the density in the EOS and produce oscillation free solutions. The extra equation being nonconservative the behavior of two established numerical schemes on shocks computation is studied and compared to exact reference solutions that are available in the present context. The analysis shows that shock conditions of the nonconservative equation have important consequence on
\end{abstract}

\footnotetext{
*Corresponding author
}

Preprint submitted to Journal of Computational Physics

July 28, 2016

(C) 2017. This manuscript version is made available under the Elsevier user license

http://www.elsevier.com/open-access/userlicense/1.0/ 
the results. Last, multidimensional computations of a supercritical gas jet is performed to illustrate the benefits of the present method, compared to conventional flow solvers.

Keywords: shock-capturing methods; real equation of state; van der Waals; nonconservative equations; pressure oscillations

\section{Introduction}

The present contribution deals with the computation of discontinuities in the Euler equations in the presence of real gas equations of state (EOS). Our considerations apply to the van der Waals (VdW) or JWL EOS [1] in their range of convexity (supercritical states for van der Waals or high-pressure detonation products for JWL). It is well known that numerical diffusion at an interface that separates two states of different densities coupled with nonlinearities in the EOS generally results in pressure variations violating interface mechanical equilibrium. This issue is not related to a loss of convexity as when dealing with phase transition and cubic EOS. This challenge is well known in the supercritical fluid community as specific solvers have been built with the aim of removing the interface problems $[2,3,4,5]$. Some of these solvers replace the energy equation by the nonconservative pressure equation. However, such a formulation is accurate for low Mach number flows, as for example in combustion, but is inaccurate when shocks are present as strict conservation of mass, momentum, and energy is mandatory to retrieve the correct propagation speed of waves. Only a few attempts are reported in the literature to cure this deficiency in the presence of both shocks and interfaces.

Starting from the work of Abgrall [6], a solution to ensure mechanical equilibrium at contact interfaces was proposed by Shyue [7]. In this approach, additional transported fields are defined, depending on the EOS, and the thermodynamics is reinterpreted in an extended way to remove the numerical difficulties alluded above. One difficulty of this technique is the nonconservative nature of the additional equations which naturally leads to concerns about the behavior of the solutions in the presence of shocks. Saurel et al. [8] considered a non-trivial projection method compared to the conventional cell volume average adopted in Godunov type schemes. Instead of computing the volume average density and energy to be used in the

EOS, Saurel et al. considered non-equilibrium states inside the cell resulting from wave propagation from boundary cells. A pressure relaxation process was used to compute the cell pressure instead of the direct use of the EOS. The method remains conservative, oscillation free, but complex and computationally expensive. Gallouet et al. [9] and Lee et al. [10] considered a hybrid method, using both conservative and primitive 
variables formulation of the Euler equations. The use of the pressure evolution equation in the primitive variables formulation enabled oscillation free computations of density discontinuities [11] but is unable to converge at shocks. The use of combined primitive and conservative formulation enables consideration of both shocks and interfaces. This is done at the cost of code complexity since two schemes have to be implemented. More recent work on a method for complex EOS is described in [12].

In the present paper a simpler alternative is proposed where the conservative formulation of the Euler equations is used everywhere. An extra evolution equation is added to the system to express the evolution of the nonlinear part of the EOS with respect to density. Doing so, the thermodynamics is slightly modified while mass, momentum and energy are strictly conserved. The modified thermodynamics is shown to converge to the results of the exact thermodynamics even in moderate cell sizes, while the conservative formulation of the Euler equations with the original thermodynamics converges to the exact solution but only with very small cells; producing spurious pressure oscillations with moderate size cells.

The method promoted in the present paper is reminiscent (and belongs to) diffuse interface methods, widely used in interface capturing with multifluid and multiphase flows [6, 13, 14]. In particular [7] and [15] considered interfaces with fluids governed by the van der Waals and Mie-Gruneisen EOS. These methods were generalized and rationalized with the help of multiphase flow modeling in the frame of liquid-gas interfaces $[16,17,18,19,20,21]$. In the present work the topic is slightly different as the medium is single phase and the volume fraction variable, that plays a key role in the formulations above, is absent.

The paper is organized as follows. In $\S 2$ the flow model is presented with its thermodynamics. For the sake of simplicity, the VdW EOS is considered only (the same analysis being valid for the JWL EOS). An example of simple transport of a contact discontinuity using conventional algorithms, resulting in severe pressure and velocity oscillations, is used to illustrate their deficiency. The analysis of the process yielding these pressure fluctuations is carried out in $\S 3$. It results in the building of the extra partial differential equation that enables the computation of an extra field variable that enters in the modified thermodynamics. With this new variable and modified EOS, pressure oscillations immediately vanish. However, the extra equation being nonconservative, some efforts have to be done to address convergence in the presence of shocks. The building of appropriate numerical scheme is done in $\S 5$. Various Riemann solvers are considered (Roe and HLLC) with different ways of inserting the nonconservative term in the Riemann solver. The influence of the intermediate state on computed results is analyzed and nonintuitive intermediate state are recommended. Last, the method is extended in two dimensions and its capabilities are illustrated on a supercritical jet simulation. 


\section{Numerical framework}

We consider the one-dimensional Euler conservation system of equations, given by

$$
\frac{\partial Q}{\partial t}+\frac{\partial F}{\partial x}=0
$$

where $Q(x, t)=\{\rho, \rho u, \rho E\}$ is the conservative vector of state, $F(Q)=\left\{\rho u, \rho u^{2}+p,(\rho E+p) u\right\}$ is the flux, $x$ denotes space and $t$ time. The quantity $E=e+u^{2} / 2$ denotes the total energy per unit mass, $e$ the internal energy, $p$ the pressure, $\rho$ the density, and $u$ the velocity. In later developments, we will refer to Eq. (1) as the original Euler system.

We assume an equally-spaced spatial grid of size $\Delta x$ for simplicity, but the generalization to non-uniform grids is straight forward following established techniques. Eq. (1) is now interpreted as being solved for the averaged conservative states in a grid cell, a finite volume method, defined by

$$
\bar{Q}_{i}(t)=\frac{1}{\Delta x} \int_{x_{i}-\Delta x / 2}^{x_{i}+\Delta x / 2} Q(x, t) d x
$$

where time is continuous for the time being. Averaging Eq. (1) according to Eq. (2), gives

$$
\frac{d \bar{Q}_{i}}{d t}+\frac{F\left(Q\left(x_{i+1 / 2}, t\right)\right)-F\left(Q\left(x_{i-1 / 2}, t\right)\right)}{\Delta x}=0 .
$$

and by approximating the flux according to

$$
F\left(Q\left(x_{i+1 / 2}, t\right)\right) \equiv F_{i+1 / 2}=\frac{1}{2}\left(F_{i}+F_{i+1}\right)-\frac{1}{2}|\tilde{M}|_{i+1 / 2} \Delta Q_{i+1 / 2},
$$

with $\Delta Q_{i+1 / 2}=\bar{Q}_{i+1}-\bar{Q}_{i}$ we arrive at the desired generic numerical method. As it is well known, different choices of $\tilde{M}$ produce different specific methods [22]. A one time-step method for Eq. (3) from $t^{n}$ to $t^{n+1}$ where $\Delta t=t^{n+1}-t^{n}$ is then used to update the numerical solution according to

$$
Q_{i}^{n+1}=Q_{i}^{n}-\frac{\Delta t}{\Delta x}\left(F_{i+1 / 2}-F_{i-1 / 2}\right)
$$

where $F_{i+1 / 2}$ is the average flux in the interval $t^{n} \leq t \leq t^{n+1}$. For convenience, we will omit the overbar over $Q$ unless it is explicitly necessary from now on. For the time being, we will consider $|\tilde{M}|_{i+1 / 2}$ given by a Roe method [23], such that $\tilde{M}_{i+1 / 2}$ obeys

$$
\Delta F_{i+1 / 2}=F_{i+1}-F_{i}=\tilde{M}_{i+1 / 2} \Delta Q_{i+1 / 2},
$$

when $\Delta Q \neq 0$ and $\tilde{M}_{i+1 / 2}$ equals the Jacobian of $F$, defined by

$$
J=\left.\frac{\partial F}{\partial Q}\right|_{Q_{i+1 / 2}},
$$


when $\Delta Q=0$. Dropping the $i+1 / 2$ subscript, for notational simplicity, the eigendecomposition $\tilde{M}=$ $R \Lambda R^{-1}$ is presumed to exist and have real eigenvalues (to ensure hyperbolicity), where $R$ denotes the matrix consisting of right eigenvectors of $\tilde{M}$ and $\Lambda$ denotes the diagonal matrix with the eigenvalues of $\tilde{M}$. Furthermore, one should also check that a complete set of eigenvectors can be found if repeated eigenvalues are present; a common occurrence in hydrodynamics. Then, the sought after matrix in Eq. (4) is defined by

$$
|\tilde{M}|=R|\Lambda| R^{-1}
$$

where the absolute value of $\Lambda$ implies the matrix with the absolute values of the diagonal elements.

The above presentation of the numerical method is well known and it is included here to ensure consistent referencing below. There are important details in the determination of $\tilde{M}$ when complex EOS are involved, since in general there is no evident or unique manner to construct $\tilde{M}$ (several approaches have been reported) to enforce Eq. (6). The equation of state considered here is the van der Waals EOS, VdW for short, given by

$$
p=\frac{(\gamma-1)\left(\rho e+a \rho^{2}\right)}{1-\rho b}-a \rho^{2},
$$

for a calorically perfect gas, with sound speed

$$
c=\sqrt{\frac{\gamma(\gamma-1)(e+a \rho)}{(1-b \rho)^{2}}-2 a \rho} .
$$

Since the basis of the present numerical method is a low-order spatial approximation with reconstructed states that are constant within each grid cell, there is no ambiguity in the meaning of the EOS. Eq. (9) applies to the states defined by $\bar{Q}$ directly since it is assumed that $Q$ is constant within each cell.

Before proceeding further, we will highlight the concrete difficulties that arise in the numerical solution of pure contact problems with complex EOS using a non-specialized solver (which will be discussed in subsequent sections). Here, we consider standard shock-capturing methods that conform to the structure of Eq. (5). The details of the calculation of $\tilde{M}$ for general EOS we use are described in [24] and they will be explained and generalized later in $\S 5.1$ and 5.2. To exemplify the issue of interest here, we consider a very simple contact problem in nitrogen $\left(\mathrm{N}_{2}\right)$, given its preponderant presence in air, which makes it inevitably involved in typical combustion of any fossil fuel. Similar examples can be found in [10]. The relevant thermodynamic data $[25,26]$ are: critical pressure of $P_{\mathrm{cr}}=33.958 \mathrm{bar}, T_{\mathrm{cr}}=126.192 \mathrm{~K}$, molecular weight $W=28.013 \mathrm{~kg} / \mathrm{kmol}$, and van der Waals coefficients $a=174.583 \mathrm{Pam}^{6} / \mathrm{kg}^{2}, b=0.0013815 \mathrm{~m}^{3} / \mathrm{kg}$ and $\gamma=1.4$. The conditions of the pure contact problem we have selected are close to the critical point of 
nitrogen, similar to the conditions considered by [3], and are given by

$$
\rho= \begin{cases}497.417 \mathrm{~kg} / \mathrm{m}^{3}(100 K) & x<0, \\ 45.9476 \mathrm{~kg} / \mathrm{m}^{3}(300 K) & 0 \leq x,\end{cases}
$$

while $u_{o}=100 \mathrm{~m} / \mathrm{s}$ and $p_{o}=40$ bars, where the domain of integration is periodic with $x \in[-1,1] \mathrm{m}$ [27]. The quantities in parenthesis in Eq. (11) indicate the corresponding temperature of these states. These conditions correspond to supercritical nitrogen fluid on both sides of the density interface. Note that although the temperature of the first state is below $T_{\mathrm{cr}}$, one can observe that in the $p-\nu$ diagram this isotherm is to the left of the critical point at the chosen pressure $p>p_{\text {cr. }}$. The exact solution of the contact problem defined by the initial condition in Eq. (11) is simply a translating contact interface to the right at constant velocity irrespective of the values of the van der Waals parameters $a$ and $b$. Note that we use actual values of the parameters of a relevant gas to highlight that the problems discussed in this paper can occur in applications of engineering importance (the issues addressed here may not appear in qualitative investigations with more benign density differences or VdW parameters that are not realistic, i.e., small). We integrated the original Euler equations using Roe and HLLC methods with a grid using 125 points and a timestep given by the Courant number (CFL) of 0.9 (similar results are obtained for smaller values of CFL and with higher-order time integration). Fig. 1 shows results of the computations at $t=4 \mathrm{~ms}$ for the ideal gas - top row (obtained by setting $a=b=0$ ) and the VdW EOS - bottom row -, as well as the exact solution. Note that the ideal and VdW EOS problems have different internal and total energy because the pressure was chosen to be the same in all cases. This results in imperceptible differences between ideal and VdW EOS on the diffused density profiles that cannot be seen in the figure. The first observation is that the density profiles shown in figure $1(\mathrm{a}, \mathrm{d})$ are diffused with respect to the exact solution, which is a well-known property of shock-capturing methods and we do not elaborate here on enhancements that can alleviate this problem; e.g., high-order methods $[28,29,30]$ or antidiffusion or compression methods [31, 32, 33]. After we accept the natural diffusion of the contact interface by this type of method, we observe that the conservative treatment of the numerical method results in the correct speed of propagation of the contact interface only for the ideal gas case. This can be inferred from the auxiliary horizontal dash-dotted line, added to figure 1(a,d), that intersects the numerical density profiles exactly at the mid point of the exact density jump. More seriously, the VdW EOS results, figure 1(d), display a shift downwards of the minimum and maximum density values. In addition, the density profile is lagging behind with respect to the exact solution. The first thought is that there is a problem of conservation with the numerical method, but that is not the case 
here. It was confirmed that the total mass, momentum, and energy were conserved to machine accuracy in these simulations and equal to $539.753 \mathrm{~kg} / \mathrm{m}^{2}, 53975.3 \mathrm{~kg} /(\mathrm{m} \mathrm{s})$, and $6.4009910^{6} \mathrm{~kg} / \mathrm{s}^{2}$, respectively. What is happening, instead, is that convergence of the VdW EOS problem (always in the sense of weak solutions of the original pde's) requires much higher resolution than when dealing with the ideal gas EOS. Discrete conservation only implies convergence of the wave speed under refinement (in the appropriate weak norm); see [34]. Increasing the number of grid points from $\mathrm{N}=125$, to 250 , to 500 , results in a minimum density value of $37.042,38.718$, and $40.3187 \mathrm{~kg} / \mathrm{m}^{3}$, respectively, at $t=4 \mathrm{~ms}$, while the expected value is 45.9476 $\mathrm{kg} / \mathrm{m}^{3}$ (similar convergence rate is observed for the maximum density and contact speed). This highlights that the convergence is very slow and it appears to require a tremendous number of grid points to achieve the same convergence exhibited by the similar contact problem using the ideal gas EOS. Evidently, a better numerical method is desired and it should not suffer from this deficiency and display faster convergence (smaller number of grid points) to the correct wave speed and preserve closer the minimum and maximum value of the density. Next, figure $1(\mathrm{~b}, \mathrm{c}, \mathrm{e}, \mathrm{f})$ show the velocity and pressure profiles at $t=4 \mathrm{~ms}$. It can be observed that the ideal gas EOS solution, figure 1(b,c), has no error in velocity or pressure, but this is not the case of the VdW EOS, figure 1(e,f). The deviation of velocity from the constant value for the VdW EOS cases is large, with a peak value close to $30 \%$ of $u_{o}$. One can show by analyzing the nonlinearity of the VdW EOS induced in the energy equation that this error is proportional to $a u_{o}$. Similarly, figure 1 (f) shows that the pressure drops by as much as $30 \%$ with respect to the initial value and now there is a clearly observed difference between the Roe and the HLLC methods. This nonlinear error manifestly pollutes what should be a simple solution and can have undesirable consequences in long-time integration and higher-dimensional problems; a well-recognized difficulty of nonideal EOS. For example, spurious pressure waves will transmit as acoustic waves that could interact with a chamber natural modes leading to erroneous predictions.

All reported weaknesses observed with the Roe solver and VdW EOS also appeared with the HLLC solver. These weaknesses are not a consequence of inappropriate linearization of the Roe matrix ([35], [36]). Here the linearization method of Toumi [24] was used, as detailed in Section 5, alleviating these issues.

In the next section, we discuss briefly the best-known approach to prevent the generation of pressurevelocity fluctuations across perfect contact interfaces and in the following section we analyze the consequences of this strategy for problems involving shocks, which includes new results that help guide the development of a new and efficient numerical method. 


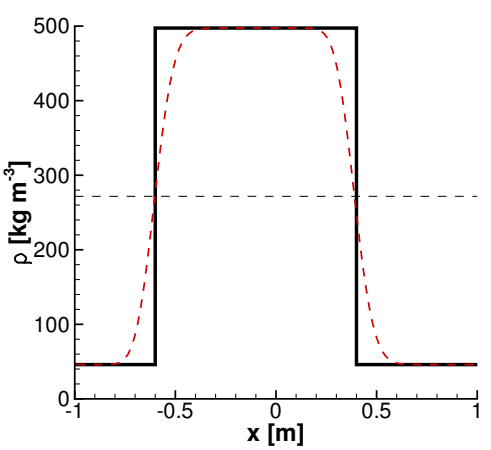

(a)

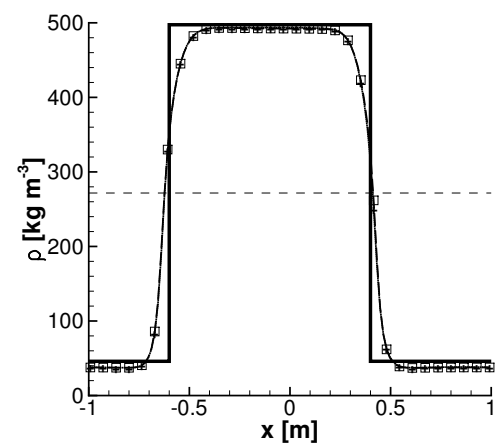

(d)

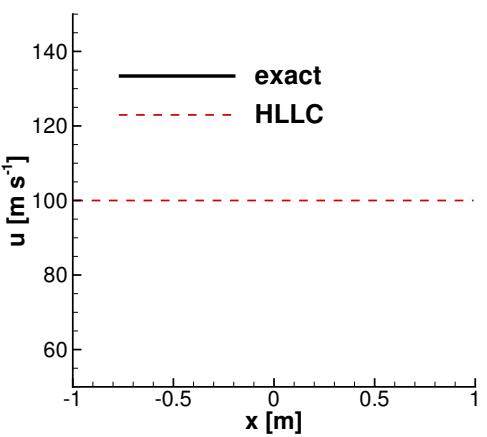

(b)

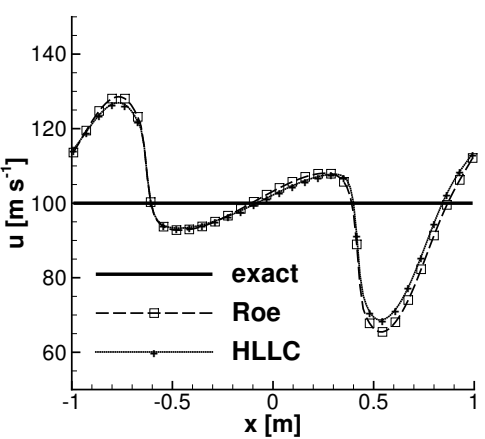

(e)

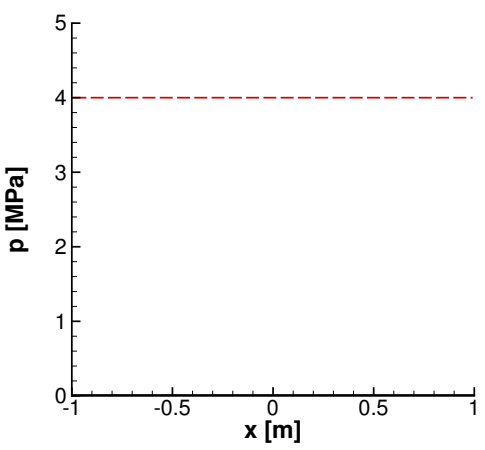

(c)

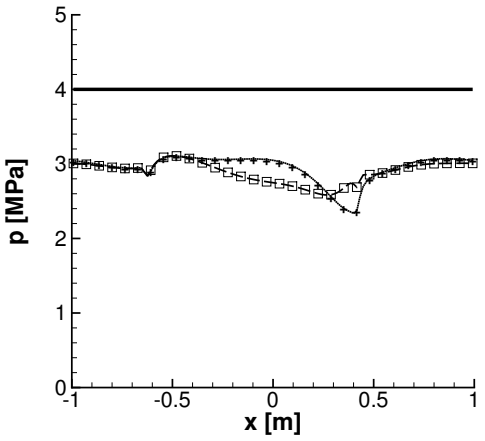

(f)

Figure 1: From left to right: density, velocity, and pressure at $t=4 \mathrm{~ms}$. Top and bottom rows correspond to ideal gas EOS and VdW EOS for $\mathrm{N}_{2}$, respectively. Black thick line denotes the exact solution. 


\section{Mechanical equilibrium}

We follow the approach described in [7] valid for the subset of EOS considered here, and write the governing equations for the contact problem where pressure and velocity are constants. The Euler equations in this situation simplify to

$$
\begin{aligned}
\frac{\partial \rho}{\partial t}+u_{o} \frac{\partial \rho}{\partial x} & =0, \\
\frac{\partial \rho e}{\partial t}+u_{o} \frac{\partial \rho e}{\partial x} & =0
\end{aligned}
$$

expressing that $\rho$ and $e$ are advected at the constant velocity $u_{o}$. To elucidate what should happen to the pressure obtained from the solution of these equations, we invert Eq. (9) and express $\rho e$ as a function of $p$ and $\rho$, according to

$$
\rho e=q p+r,
$$

where

$$
q=\frac{(1-b \rho)}{\gamma-1}, \quad r=a \frac{(2-\gamma-b \rho)}{\gamma-1} \rho^{2},
$$

and introduce Eq. (14) in Eq. (13). From this point onwards, we refer to the functions defined by Eq. (15) as $q(\rho)$ and $r(\rho)$; the explicit presence of the argument $\rho$ being the indication that we are referring to these functions. After application of the chain rule, we obtain

$$
q\left(\frac{\partial p}{\partial t}+u_{o} \frac{\partial p}{\partial x}\right)+p\left(\frac{\partial q}{\partial t}+u_{o} \frac{\partial q}{\partial x}\right)+\left(\frac{\partial r}{\partial t}+u_{o} \frac{\partial r}{\partial x}\right)=0 .
$$

It is important to note that the chain rule cannot be applied inside terms containing functions of $\rho$ in Eq. (16), e.g.: $q(\rho)$ or $r(\rho)$ in Eq. (14). If $p=p_{o}$ is constant, the requirement of mechanical equilibrium is satisfied as long as

$$
\begin{aligned}
& \frac{\partial q}{\partial t}+u_{o} \frac{\partial q}{\partial x}=0 \\
& \frac{\partial r}{\partial t}+u_{o} \frac{\partial r}{\partial x}=0
\end{aligned}
$$

These equations are a subset of those already identified in [7] and used to construct a formulation compatible with mixtures of nonideal EOS (van der Waals and stiffened EOS). Here, we will concentrate on a "minimal" formulation for a pure $\mathrm{VdW}$ EOS, assuming $\gamma, a$ and $b$ are constants but concentrating on the details of the solutions of the new extended systems, which have not been studied previously. This will help us clarify some of the consequences of Eqs. (17)-(18) and the implications of their use. 
One can easily see that thanks to Eq. (12), Eq. (17) is satisfied identically because $q(\rho)$ is linear on $\rho$. Next, we can develop $r(\rho)$ into two terms, one proportional to $\rho^{2}$ and a second term proportional to $\rho^{3}$, since

$$
r=\frac{a \rho^{2}}{\gamma-1}(2-\gamma-b \rho)=\left(a \frac{2-\gamma}{\gamma-1}\right) \rho^{2}-\left(\frac{a b}{\gamma-1}\right) \rho^{3} .
$$

Therefore, mechanical equilibrium is satisfied as long as either Eq. (18) or

$$
\frac{\partial \rho^{2}}{\partial t}+u_{o} \frac{\partial \rho^{2}}{\partial x}=0, \quad \text { and } \quad \frac{\partial \rho^{3}}{\partial t}+u_{o} \frac{\partial \rho^{3}}{\partial x}=0
$$

are enforced. Solving an equation for $r$ alone or two equations for $\rho^{2}$ and $\rho^{3}$ and then combining the result according to Eq. (19) will ensure mechanical equilibrium. Evidently, the first choice is minimal, just one extra equation needs to be solved and it may look superfluous to consider Eq. (20) further. But, as discussed next, it is not entirely evident how to transform Eq. (18) into an equation for $r$ that is applicable to flows with shock waves or when $u$ is not constant.

To obtain the consistent equation, which was derived by [7] without a detailed explanation, we turn to Eq. (20) which are valid for constant $u$. We seek the more general equations that degenerate into Eq. (20) when the velocity is constant, and at the same time remain exactly compatible with the mass conservation equation, Eq. (1). In other terms, we need to derive the equations for $\rho^{2}$ and $\rho^{3}$ from the primitive equation for $\rho$. This is easily done by multiplying the mass-conservation equation by $2 \rho$ and $3 \rho^{2}$, respectively, to arrive at the quasi-conservation forms given by

$$
\frac{\partial \rho^{2}}{\partial t}+\frac{\partial\left(u \rho^{2}\right)}{\partial x}+\rho^{2} \frac{\partial u}{\partial x}=0, \quad \text { and } \quad \frac{\partial \rho^{3}}{\partial t}+\frac{\partial\left(u \rho^{3}\right)}{\partial x}+2 \rho^{3} \frac{\partial u}{\partial x}=0 .
$$

It is evident that these equations degenerate into Eq. (20) when $u$ is constant, and therefore they are suitable for addressing the mechanical equilibrium problem while preserving compatibility with the original nonlinear equations (which is important in the presence of shocks). We can now use Eq. (21) and the definition of Eq. (19) to arrive at a single equation for $r$, given by

$$
\frac{\partial r}{\partial t}+\frac{\partial(u r)}{\partial x}+\left(r-\kappa \rho^{3}\right) \frac{\partial u}{\partial x}=0
$$

where $\kappa=a b /(\gamma-1)$.

\section{Equivalence between the original and extended systems}

In [7], focus was directed mainly at the derivation of mechanical-equilibrium compatible equations based on generalization of previous ideas $[6,13,14]$. Here, we elaborate on important implications of the strategy 
introduced in those papers. The basic idea implied by the annexation of Eq. (22) to the original Euler system is the extension of the thermodynamical EOS. The new "extended" EOS is also a function of $r$, in addition of $\rho e$ and $\rho$, and it is defined by

$$
p=\Gamma(\rho)(\rho e-r),
$$

where

$$
\Gamma=\frac{1}{q(\rho)}=\frac{\gamma-1}{1-b \rho}
$$

is the Gruneisen coefficient with $q$ defined by Eq. (15). The system comprising the original Euler equations with Eq. (22) and Eq. (23) is termed from this point onwards as the extended Euler equations. Of course, EOS consistency requires that Eq. (23) reduces to the original Eq. (9) when it is projected to the manifold defined by Eq. (19). The strategy developed in [7] opens some new avenues for the design of numerical methods because the extra field in Eq. (23), $r$, can be used to ensure mechanical equilibrium in pure contact problems (a notion of nonlinear numerical stability). But, one important question that was not investigated in [7] is whether solutions of the original Euler system are equivalent to solutions obtained from the extended Euler system.

Solutions from the two systems are certainly equivalent if they are sufficiently smooth, in which case we do not need to be concerned anymore. But, we must be careful if the solution is not smooth because $r$ may not converge under grid refinement to $r(\rho)$. According to well-understood hyperbolic equations theory $[37,38]$, the speed of propagation of discontinuities is uniquely defined from the Rankine-Hugoniot jump relations if the system is in conservation form (there may be selection process to determine which wave is the physically correct one, but the speed of the wave itself is uniquely defined). Furthermore, the speed of discontinuous waves will converge to the correct value as long as the numerical method is written in discretely conservative form [34]. Now, these statements do not apply to Eq. (22) because the equation is not in conservation form and the speed of $r$-waves (for lack of a better term) generally depends on additional information that must be provided [39]. There is no guaranty that the speed of $r$-waves will converge to the speed of the contact. When that happens, the extended EOS will no longer reduce to the original EOS. Therefore, additional care must be employed when approximating numerically Eq. (22). In fact, this is the price to pay when introducing Eq. (23) and extending the system of equations. The rest of the paper is aimed at the construction of numerical methods that ensure that $r$-waves and contacts have the same (or very close) speed. 


\section{Numerical method for the extended system in non-conservation form}

We now present the construction of a numerical method for the quasi-conservation "extended" system of equations, that includes Eq. (22), and that we cast in the form

$$
\frac{\partial Q}{\partial t}+\frac{\partial F}{\partial x}+A \frac{\partial Q}{\partial x}=0
$$

where $Q(x, t)=\{\rho, \rho u, \rho E, r\}$ is the vector of state and $F(Q)=\left\{\rho u, \rho u^{2}+p,(\rho E+p) u, u r\right\}$ is the flux, and

$$
A=\left[\begin{array}{cccc}
0 & 0 & 0 & 0 \\
0 & 0 & 0 & 0 \\
0 & 0 & 0 & 0 \\
-\beta u & \beta & 0 & 0
\end{array}\right],
$$

contains the non-conservation form coefficients, where

$$
\beta=\frac{r}{\rho}-\kappa \rho^{2}
$$

The main difficulty in the construction of a numerical method for Eq. (25) is the interpretation of the non-conservation form term, some times referred to as the nonconservative product, in the presence of discontinuities because the theory of distributions cannot give a meaning to this term. To address this problem, we chose to employ the generalization for nonconservative products due to [40]. In this theory, one considers a family of smooth mapping $\Phi(s)$ connecting a discontinuity in the vector of state (a Riemann problem) such that

$$
Q_{L}=\Phi\left(0 ; Q_{L}, Q_{R}\right), \quad Q_{R}=\Phi\left(1 ; Q_{L}, Q_{R}\right),
$$

where $Q_{L}$ and $Q_{R}$ denote left and right sates, respectively. The function $\Phi(s)$ satisfies

$$
\left|\frac{\partial \Phi}{\partial s}\left(s ; Q_{L}, Q_{R}\right)\right| \leq K_{1}\left|Q_{L}-Q_{R}\right|
$$

where

$$
\left|\frac{\partial \Phi}{\partial s}\left(s ; Q_{L}, Q_{R}\right)-\frac{\partial \Phi}{\partial s}\left(s ; Q_{L}^{\prime}, Q_{R}^{\prime}\right)\right| \leq K_{2}\left(\left|Q_{L}-Q_{R}\right|+\left|Q_{L}^{\prime}-Q_{R}^{\prime}\right|\right),
$$

for all $s$, where $K_{1}$ and $K_{2}$ are constants, independent of $Q_{L}, Q_{R}, Q_{L}^{\prime}$ and $Q_{R}^{\prime}$. With recourse to Eq. (7), now defined as

$$
J=\frac{\partial F}{\partial Q}+A
$$


we interpret the matrix $\tilde{M}$ in Eq. (6) as the solution of

$$
\int_{0}^{1} J\left(\Phi\left(s ; Q_{L}, Q_{R}\right)\right) \frac{\partial \Phi}{\partial s}\left(s ; Q_{L}, Q_{R}\right) d s=\tilde{M} \Delta Q
$$

where $\Delta Q=Q_{R}-Q_{L}$, and it is omitted in the notation (solely for convenience) that $\tilde{M}$ depends on the choice of $\Phi$. Note that, as it is well known, a numerical method based on Eqs. (4)-(5) is not applicable here and it will have to adopt a different form for non-conservation-form systems; something that is done in $\S 5.1$.

At this stage, many simple forms of $\Phi$ could be used directly to construct usable matrices $\tilde{M}$. Furthermore, for each choice of $\Phi$ it is, in principle, possible to define further numerical families by approximating the quadrature in Eq. (32) to different orders of accuracy; although in practice the exact quadratures tend to perform better and we will adhere to this practice whenever possible. Some of the well-known choices of $\Phi$ include

$$
\Phi=Q_{L}+s\left(Q_{R}-Q_{L}\right),
$$

[41], van Leer's method where $\tilde{M}=J(\bar{Q})$ with $\bar{Q}=\frac{1}{2}\left(Q_{L}+Q_{R}\right)$, and the weighted mapped states of [24]. The later is a quite general approach since it enables one to recover the exact Roe formulation for the original Euler system (where a flux exists) without ambiguities. In that formulation, we chose $\Phi$ through an intermediate vector of state, $W$, that is an invertible smooth map, $Q_{o}(W)$, such that $Q=Q_{o}(W)$ and $W=W_{o}(Q)$ are unique, and define

$$
\Phi\left(s ; Q_{L}, Q_{R}\right)=Q_{o}(W(s)),
$$

where $W(s)=W_{L}+s\left(W_{R}-W_{L}\right)$ is a linear path analogous to Eq. (33). We now write Eq. (32) as

$$
\tilde{C} \Delta W=\tilde{M} \Delta Q
$$

where

$$
\tilde{C}=\int_{0}^{1} J\left(Q_{o}(W(s))\right) \frac{\partial Q_{o}}{\partial W}(W(s)) d s
$$

and

$$
\Delta Q=\tilde{B} \Delta W
$$

with

$$
\tilde{B}=\int_{0}^{1} \frac{\partial Q_{o}}{\partial W}(W(s)) d s .
$$

Introducing Eq. (37) in Eq. (35), results in

$$
\tilde{C} \Delta W=\tilde{M} \tilde{B} \Delta W
$$


and finally, from Eq. (39) we deduce

$$
\tilde{M}=\tilde{C} \tilde{B}^{-1} .
$$

The choice of $Q_{o}$ defines the particular integration path, $\Phi$, and indirectly the corresponding averaging. Roe [23] and Toumi showed that for the original Euler equations one can chose $W=\sqrt{\rho}(1, u, H)$, where $H=E+p / \rho$, to recover exactly the Roe-average states for ideal gases. Toumi [24] also used the same mapping to derive a unique Roe matrix for a general EOS. It is this approach that was used to formulate the Roe method used in the results presented in figure 1.

Now, we extend [24] to the non-conservation system of the form given by Eq. (25). This requires a choice for $W$ that produces a consistent treatment for $r$. After some considerations that will be explained immediately, it becomes clear that $W$ should be chosen as

$$
W=\left(w_{1}, w_{2}, w_{3}, w_{4}\right)=\{\sqrt{\rho}, \sqrt{\rho} u, \sqrt{\rho} H, r / \sqrt{\rho}\} .
$$

In [24], the deformation of the integration path is dictated exclusively by the transformation Eq. (41), since the dependence of $\Phi$ on $s$ is completely specified once $W$ is chosen. One can verify, below, that Eq. (41) gives perfect matching of the contact and $r$-waves speeds for pure contact problems and it also gives the correct speeds for shocks in the asymptotic limit of small $a$, and for these reasons this mapping was chosen from this point onwards.

We did consider a general mapping of the form $r=w_{1} f\left(w_{4}\right)$ that lead to a differential equation with solution $f=a_{0}+a_{1} \exp \left(a_{2} w_{4}\right)$. Unfortunately, after carrying out all the algebraic manipulations explained in Appendix B, one finds that there is no solution for the parameters $a_{0}, a_{1}$, and $a_{2}$ that ensures a matching of the $r$-wave and contact speeds exactly (as desired from the equivalence concept in $\S 4$ ). We learned from this attempt that it appears that at least one must consider $W$ mappings of the form $\rho=f\left(w_{1}, w_{4}\right)$ and $r=g\left(w_{1}, w_{4}\right)$ if exact matching of speeds is desired. This would make the analytical determination of $\tilde{M}$ impossible and therefore was not pursued. But, as will be shown shortly, the choice in Eq. (41) will turn out to be useful in the end.

\subsection{Extended Roe-based method}

The numerical method given in Eq. (5) is not appropriate for Eq. (25) because our system cannot be written in conservation form. Therefore, one rewrites the method in streamwise-difference form [22] (also known as the fluctuation form), as follows

$$
Q_{i}^{n+1}=Q_{i}^{n}-\frac{\Delta t}{\Delta x}\left[\tilde{M}_{i-1 / 2}^{+}\left(Q_{i}-Q_{i-1}\right)+\tilde{M}_{i+1 / 2}^{-}\left(Q_{i+1}-Q_{i}\right)\right],
$$


where the decomposition based on the eigensystem of $\tilde{M}$, analogous to Eq. (8), defines

$$
\tilde{M}^{ \pm}=R \Lambda^{ \pm} R^{-1}
$$

where

$$
\Lambda^{ \pm}=\frac{\Lambda \pm|\Lambda|}{2} .
$$

The subscript $i \pm 1 / 2$ has been dropped here for simplicity of notation, as it was done before. The form of the method in Eq. (42), which is exactly equivalent to the flux form Eq. (5) for a conservation-form system, is applicable to the quasi-conservation form Eq. (25), after a proper definition of $\tilde{M}$ is chosen, as explained above.

The lengthy calculation in Appendix B results in the following Roe-averaged matrix for our extended system

$$
\tilde{M}=\left[\begin{array}{cccc}
0 & 1 & 0 & 0 \\
\frac{1}{2} \hat{u}^{2}(\tilde{\Gamma}-2)+\tilde{\Gamma} \hat{\alpha} & (2-\tilde{\Gamma}) \hat{u} & \tilde{\Gamma} & -\tilde{\Gamma} \\
\left(\tilde{\Gamma}\left(\hat{\alpha}+\frac{\hat{u}^{2}}{2}\right)-\hat{H}\right) \hat{u} & \hat{H}-\tilde{\Gamma} \hat{u}^{2} & (\tilde{\Gamma}+1) \hat{u} & -\tilde{\Gamma} \hat{u} \\
\frac{1}{2}\left[\kappa \tilde{\alpha}_{2}-\tilde{\phi}+\hat{u}\left(\kappa \tilde{\alpha}_{1}-3 \hat{\omega}\right)\right] & 2 \hat{\omega}-\kappa \tilde{\alpha}_{1} & 0 & \hat{u}
\end{array}\right],
$$

where

$$
\begin{gathered}
\hat{u}=\frac{\sqrt{\rho_{L}} u_{L}+\sqrt{\rho_{R}} u_{R}}{\sqrt{\rho_{L}}+\sqrt{\rho_{R}}}, \quad \hat{H}=\frac{\sqrt{\rho_{L}} H_{L}+\sqrt{\rho_{R}} H_{R}}{\sqrt{\rho_{L}}+\sqrt{\rho_{R}}}, \quad \hat{\omega}=\frac{r_{L} / \sqrt{\rho_{L}}+r_{R} / \sqrt{\rho_{R}}}{\sqrt{\rho_{L}}+\sqrt{\rho_{R}}}, \\
\tilde{\alpha}_{1}=\frac{\int_{0}^{1} w_{1}^{5}(s) d s}{\frac{1}{2}\left(\sqrt{\rho_{L}}+\sqrt{\rho_{R}}\right)}, \quad \tilde{\alpha}_{2}=\frac{\int_{0}^{1} w_{2}(s) w_{1}^{4}(s) d s}{\frac{1}{2}\left(\sqrt{\rho_{L}}+\sqrt{\rho_{R}}\right)}, \quad \tilde{\phi}=\frac{\int_{0}^{1} w_{2}(s) w_{4}(s) / w_{1}(s) d s}{\frac{1}{2}\left(\sqrt{\rho_{L}}+\sqrt{\rho_{R}}\right)},
\end{gathered}
$$

with $L$ and $R$ subscripts denoting left and right states, respectively, i.e., $i$ and $i+1$ cell values when determining $\tilde{M}_{i+1 / 2}$. Exact expressions for the quantities in Eq. (47) are given in Eqs. (B.19)-(B.22). The averaged Gruniesen coefficient is denoted by $\tilde{\Gamma}$, and it is defined by

$$
\tilde{\Gamma}=\int_{0}^{1} \Gamma\left(w_{1}(s)\right) d s
$$

while $\hat{\alpha}$ is defined in Eq. (B.33) and $w_{k}(s)$ are given by Eq. (41). As shown in the appendix, $\tilde{\alpha}_{1}, \tilde{\alpha}_{2}$ and $\tilde{\phi}$ can be calculated analytically, and only Eq. (48) requires numerical quadrature. This integral was obtained by using 4-point Gauss-Legendre quadrature, which is accurate for polynomials up to degree 7.

The next step in the analysis of the numerical method is to ensure that the spectrum of Eq. (45) is purely real (hyperbolic). First, let us consider the situation for which the methodology was motivated, i.e., the perfect solution of contacts without spurious pressure or velocity fluctuations, advected at the constant 
velocity $u_{o}$. From the definitions of the different parameters we have $\hat{u}=u_{o}, \tilde{\alpha}_{2}=u_{o} \tilde{\alpha}_{1}, \phi_{2}=u_{o} \hat{\omega}$, and one can determine the following eigenspeeds, $\left\{u_{o}-c_{o}, u_{o}, u_{o}, u_{o}+c_{o}\right\}$, where

$$
c_{o}^{2}=\tilde{\Gamma}\left(\hat{H}-2 \hat{\omega}-\frac{\hat{u}^{2}}{2}+\hat{\alpha}+\kappa \tilde{\alpha}_{1}\right) .
$$

Clearly, the contact and $r$-waves are perfectly compatible because the eigenvalue $u_{o}$ is repeated twice and there would never be a divergence (splitting) of the two types of waves (as long as Eq. (49) is positive). This implies long-time equivalence of the extended EOS approach with the original Euler system; which is the desired property.

In the general situation, not a pure contact, the spectrum of Eq. (45) can also be determined analytically. It corresponds to the eigenspeeds $\left\{\hat{u}-\hat{c}^{-}, \hat{u}, \hat{u}+\delta \hat{c}, \hat{u}+\hat{c}^{+}\right\}$, where

$$
\begin{aligned}
\delta \hat{c} & =\frac{1}{4} \sqrt{8 c_{o}^{2}-\frac{482^{2 / 3} c_{o}^{4}}{Y^{2 / 3}}-\frac{Y^{2 / 3}}{32^{2 / 3}}}-\frac{\sqrt[3]{2} c_{o}^{2}}{\sqrt[3]{Y}}-\frac{\sqrt[3]{Y}}{12 \sqrt[3]{2}}, \\
\hat{c}^{+} & =\frac{2 \sqrt[3]{2} c_{o}^{2}}{\sqrt[3]{Y}+\frac{\sqrt[3]{Y}}{6 \sqrt[3]{2}}}, \\
\hat{c}^{-} & =\frac{1}{4} \sqrt{8 c_{o}^{2}-\frac{482^{2 / 3} c_{o}^{4}}{Y^{2 / 3}}-\frac{Y^{2 / 3}}{32^{2 / 3}}}+\frac{\sqrt[3]{2} c_{o}^{2}}{\sqrt[3]{Y}}+\frac{\sqrt[3]{Y}}{12 \sqrt[3]{2}},
\end{aligned}
$$

with $c_{o}$ defined by Eq. (49) and

$$
Y=108 \Delta+\sqrt{432\left(27 \Delta^{2}-16 c_{o}^{6}\right)}
$$

with

$$
\Delta=\tilde{\Gamma}\left[(\tilde{\phi}-\hat{u} \hat{\omega})-\kappa\left(\tilde{\alpha}_{2}-\hat{u} \tilde{\alpha}_{1}\right)\right]
$$

The two intermediate eigenvalues $\{\hat{u}, \hat{u}+\delta \hat{c}\}$ correspond to the speeds of the new $r$-wave and the intermediate contact, respectively, and they are not identical; $\delta \hat{c}$ being the speed mismatch. This has further implications because it also implies that the states behind a shock will not ensure Eq. (19) and we will lose exact equivalence, as defined in $\S 4$. The error is not large in general and one can estimate its magnitude by carrying out an expansion of the eigenvalues for $\Delta / c_{o}^{3} \ll 1$, which gives

$$
\begin{aligned}
\delta \hat{c} & =-\frac{\Delta}{2 c_{o}^{2}}+O\left(\Delta^{3}\right), \\
\hat{c}^{ \pm} & =c_{o} \pm \frac{\Delta}{4 c_{o}^{2}}-\frac{3}{32} \frac{\Delta^{2}}{c_{o}^{5}}+O\left(\Delta^{3}\right) .
\end{aligned}
$$

$\delta \hat{c} / c_{o}$ is typically very small because $\Delta$, defined in Eq. (54), represents the difference of terms that are of the same kind, and it vanishes identically for a contact. These properties are some of the nice consequences 
of using the systematic method in [24] to construct the averaged matrix $\tilde{M}$, which we have applied here to a non-conservation form system.

One can verify that in the case of a simple contact we have $\Delta=0$ and the equations produce the roots listed in the previous paragraph, Eq. (49); although $Y$ becomes purely imaginary in this case, the roots are real when replaced into Eqs. (50)-(52) and one finds $\hat{c}^{+}=\hat{c}^{-}$and $\delta \hat{c}=0$ as expected.

The eigenvectors of $\tilde{M}$, Eq. (45), up to first order in $\Delta$ and ordered according to the eigenvalues presented before are given by

$$
R_{1}=\left[\begin{array}{c}
1 \\
\hat{u}-c_{o}+\frac{\Delta}{4 c_{o}^{2}} \\
\hat{H}-c_{o} \hat{u}+\frac{\hat{u} \Delta}{4 c_{o}^{2}} \\
2 \hat{\omega}-\kappa \tilde{\alpha}_{1}+\frac{\Delta}{2 \tilde{\Gamma} c_{o}}
\end{array}\right], \quad R_{2}=\left[\begin{array}{c}
-1 \\
-\hat{u}+\frac{\Delta}{2 c_{o}^{2}} \\
\frac{\hat{u} \Delta}{2 c_{o}^{2}} \\
\frac{1}{2} \hat{u}^{2}-\hat{\alpha}
\end{array}\right], \quad R_{3}=\left[\begin{array}{c}
1 \\
\hat{u}-\frac{\Delta}{2 c_{o}^{2}} \\
\frac{1}{2} \hat{u}^{2}-\hat{\alpha}-\frac{\hat{u} \Delta}{2 c_{o}^{2}} \\
0
\end{array}\right], \quad R_{4}=\left[\begin{array}{c}
1 \\
\hat{u}+c_{o}+\frac{\Delta}{4 c_{o}^{2}} \\
\hat{H}+c_{o} \hat{u}+\frac{\hat{u} \Delta}{4 c_{o}^{2}} \\
2 \hat{\omega}-\kappa \tilde{\alpha}_{1}-\frac{\Delta}{2 \tilde{\Gamma} c_{o}}
\end{array}\right] .
$$

One advantage of the approach followed in this section is that we have determined the path-averaged matrix analytically. This, in turn, allowed us to find the spectrum of the system, to ensure hyperbolicity of the averaged matrix, and to verify the impact of the nonconservative path selection on the characteristic speeds of the system. As can be seen, the chosen path $\Phi$, implicitly selected by the choice of mapping in Eq. (41), has an impact in the wave structure. Ideally, one would like to chose $W$ such that $\delta \hat{c}$ is identically zero (exact matching of contact and $r$-wave speeds), but evidently the nonlinearity of the equation of state makes this goal very difficulty to achieve. Nevertheless, $\delta \hat{c}$ is very small; identically zero for a pure contact situation. Our approach contrasts with the common practice in the construction of numerical methods for non-conservation form systems, where Eq. (32) is invariably determined by recurring to a numerical quadrature typically using the path defined by Eq. (33). While this simplifies the formulation of the numerical method, it carries the danger of hiding undesirable consequences for non-smooth problems, i.e., with shocks, since one cannot know beforehand by how much are the different speeds of the system altered by the choice of path of integration; an observation that has been highlighted by [42]. Furthermore, the use of the path defined by Eq. (33) precludes an analytical derivation of the averaged matrix, something that it is easy to verify, rendering the method more computationally expensive than that proposed here.

The performance of Eq. (42) with Eq. (45) will be evaluated in $\S 5.3$ with contact and shock problems and the results will be discussed in detail there. But, it is well recognized that while the Roe method is very elegant, it is not always the most efficient numerical strategy for complex problems. In this section, 
we developed the generalize Roe method because it contains elements required in the next section for the formulation of the more efficient generalized HLLC method.

\subsection{Extended HLLC-based method}

Here, we generalize the well-known HLLC method [43] to the non-conservation form system given in Eq. (25). The starting point is integration of the governing equations, giving

$$
Q_{i}^{n+1}=Q_{i}^{n}-\frac{\Delta t}{\Delta x}\left(F_{i+1 / 2}-F_{i-1 / 2}\right)-\frac{1}{\Delta x} \int_{t_{n}}^{t_{n+1}} \int_{x_{i-1 / 2}}^{x_{i+1 / 2}} A \frac{\partial Q}{\partial x} d x d t
$$

where integration by parts of the flux term has been used and the non-conservation product integral term remains to be determined explicitly somehow. The cell-face fluxes, $F_{i \pm 1 / 2}$, are determined according to the well-known procedure. First, we assume the left and right wave speeds, $S_{L}$ and $S_{R}$, of the underlying Riemann problem at a given cell face $i+1 / 2$ are known. These speeds can be determined easily now from the results of the previous section as $\hat{u}-\hat{c}^{-}$and $\hat{u}+\hat{c}^{+}$, respectively, from Eqs. (51)-(52). The advantage of using these values is that they consistently incorporate many details of the nonideal EOS, as well as the interactions with the extra equation for $r$. In our tests of the method to be described later, we find that using directly Eq. (49) as the speed estimate gives results that are indistinguishable from those obtained with the generalized Roe method using the full system discussed in the previous section. Now, choosing appropriate paths of integration one can determine the following flux relations, as explained in [43], for the conservative part of Eq. (58), according to

$$
F_{K}^{*}=F_{K}+S_{K}\left(Q_{K}^{*}-Q_{K}\right)
$$

where $K=L$ or $R$ and $F_{K}=F\left(Q_{K}\right)$, and the intermediate state is given by

$$
Q_{K}^{*}=\frac{S_{K}-u_{K}}{S_{K}-S_{M}}\left[\begin{array}{c}
\rho_{K} \\
\rho_{K} S_{M} \\
(\rho E)_{K}+\left(S_{M}-u_{K}\right)\left(\rho_{K} S_{M}+\frac{p_{K}}{S_{K}-u_{K}}\right) \\
r_{K}
\end{array}\right]
$$

and for later reference we have

$$
r_{K}^{*}=\frac{S_{K}-u_{K}}{S_{K}-S_{M}} r_{K}
$$

Above, the speed of the intermediate wave of this simplified Riemann problem $u^{*}=S_{M}$ is given by

$$
S_{M}=\frac{p_{R}-p_{L}+\rho_{L} u_{L}\left(S_{L}-u_{L}\right)-\rho_{R} u_{R}\left(S_{R}-u_{R}\right)}{\rho_{L}\left(S_{L}-u_{L}\right)-\rho_{R}\left(S_{R}-u_{R}\right)} .
$$


For the $L$ state given by $Q_{i}$ and the $R$ state given by $Q_{i+1}$, the flux is then determined according to

$$
F_{i+1 / 2}= \begin{cases}F_{L} & S_{L}>0, \\ F_{L}^{*} & S_{L}<0<S_{M}<S_{R}, \\ F_{R}^{*} & S_{L}<S_{M}<0<S_{R}, \\ F_{R} & S_{R}<0 .\end{cases}
$$

The novelty here is in the determination of the nonconservative product, last term in Eq. (58). To remain consistent with the HLLC strategy, we assume the same underlying Riemann problem composed of three waves and that the states are constant within the wave fronts with states given by the HLLC formulas. We can then see that the integrand in the last term of Eq. (58) is only non-zero across the wave fronts, but the integral is impossible to define uniquely without further knowledge. To resolve this problem and be able to compute the quadrature, we employ the integration path strategy described in $\S 5$ by observing that the only non-zero contributions to these integrals come from the left and right waves that emanate from the cell walls and propagate into the cells. There can be up to four such waves with speeds $S_{L, i \pm 1 / 2}$ and $S_{R, i \pm 1 / 2}$, respectively, whose graph might be contained within cell $i$. The intermediate contact wave of the approximate Riemann problems do not contribute to the quadrature because the velocity is constant through the contact wave and the last term in Eq. (25) is therefore zero. Introducing the path described in Eq. (41) we retrieve for each wave contribution to the last integral in Eq. (58), the following

$$
\frac{1}{\Delta x} \int_{t_{n}}^{t_{n+1}} f_{0}^{1} A \frac{\partial Q}{\partial W} \frac{\partial W}{\partial x} d x d t=\frac{\Delta t}{\Delta x} f_{0}^{1} A \frac{\partial Q}{\partial W} \frac{\partial W}{\partial s} d s=\frac{\Delta t}{\Delta x}\left(f_{0}^{1} A \frac{\partial Q}{\partial W} d s\right) \Delta W,
$$

where $f$ indicates that the integral is carried out only across the wave thickness. Introducing the definition of $A$, we have for each of the waves of the form given in Eq. (64), the wave integrals

$$
\begin{aligned}
\left(f_{0}^{1} A \frac{\partial Q}{\partial W} d s\right) \Delta W & \equiv \int_{0}^{1}\left(r-\kappa \rho^{3}\right) \frac{\partial u}{\partial s} d s=\int_{0}^{1}\left(r-\kappa \rho^{3}\right) \frac{\partial}{\partial s}\left(w_{2} / w_{1}\right) d s \\
& =\int_{0}^{1}\left(w_{1}(s) w_{4}(s)-\kappa w_{1}^{6}(s)\right)\left(\frac{\Delta w_{2}}{w_{1}(s)}-\frac{w_{2}(s)}{w_{1}^{2}(s)} \Delta w_{1}\right) d s \\
& =\int_{0}^{1}\left(w_{4}-\kappa w_{1}^{5}\right)\left(\Delta w_{2}-\frac{w_{2}}{w_{1}} \Delta w_{1}\right) d s \\
& =\left[\left(\kappa \tilde{\alpha}_{2}-\tilde{\phi}\right) \Delta w_{1}+\left(\hat{\omega}-\kappa \tilde{\alpha}_{1}\right) \Delta w_{2}\right] \bar{w}_{1},
\end{aligned}
$$

where $\tilde{\alpha}_{1}, \tilde{\alpha}_{2}$, and $\tilde{\phi}$ were defined in Eq. (47). The states that are implied in Eq. (65) are $W_{L}$ and $W_{L}^{*}$ for the $L$ wave (moving at speed $S_{L}$ ) i.e. $W(s)=W_{L}+\left(W_{L}^{*}-W_{L}\right) s$, and $W_{R}^{*}$ and $W_{R}$ for the $R$ wave (moving 


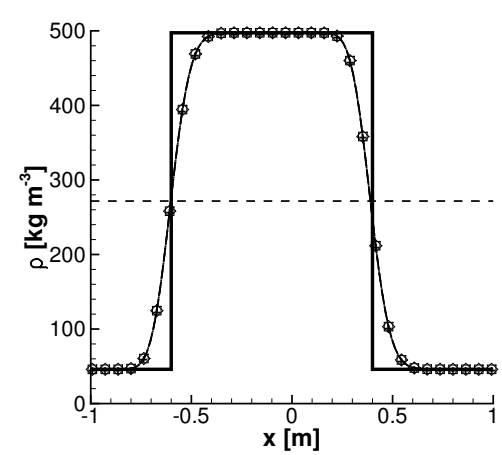

(a)

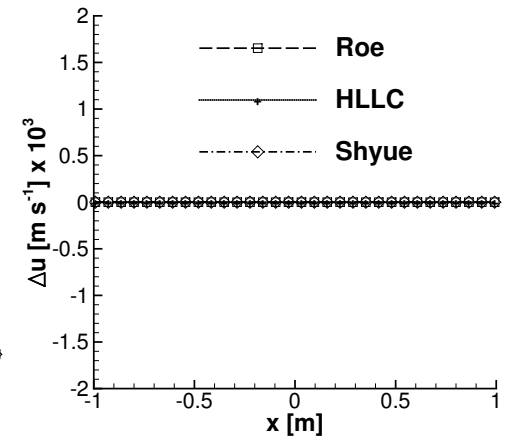

(b)

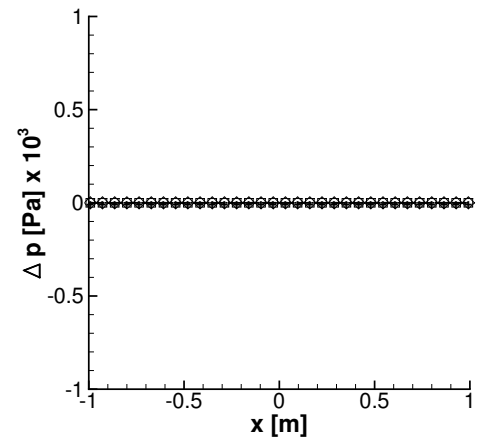

(c)

Figure 2: From left to right: density, velocity, and pressure at $t=4 \mathrm{~ms}$. Black thick line denotes the exact solution of density at this time.

at speed $\left.S_{R}\right)$ i.e. $W(s)=W_{R}^{*}+\left(W_{R}-W_{R}^{*}\right) s$. These $W$ states are obtained from the states $Q_{L}, Q_{L}^{*}, Q_{R}^{*}$ and $Q_{R}$, respectively. To complete the algorithm, one must chose $w_{L, 4}^{*}$ and $w_{R, 4}^{*}$ in the path integrals Eq. (65). The following possibilities are considered here:

- HLLC(a): One possibility is to use $r_{L}^{*}$ or $r_{R}^{*}$, defined by Eq. (61), such that $w_{L, 4}^{*}=r_{L}^{*} / \sqrt{\rho_{L}^{*}}$ and $w_{R, 4}^{*}=r_{R}^{*} / \sqrt{\rho_{R}^{*}}$, respectively.

- $\mathrm{HLLC}(\mathrm{b})$ : Another possibility is to select $w_{L, 4}^{*}=r\left(\rho_{L}^{*}\right) / \sqrt{\rho_{L}^{*}}$ and $w_{R, 4}^{*}=r\left(\rho_{R}^{*}\right) / \sqrt{\rho_{R}^{*}}$, using Eq. (19).

Finally, when we consider the physical process that is presumed to take place within each grid cell, it is clear that in order for Eq. (65) to produce unambiguous results there cannot be any interactions between the wave patterns from the left and right cell faces, i.e., $i \pm 1 / 2$. This implies that the CFL stability condition cannot exceed $1 / 2$, which is more restrictive than that implied by the conservative part of the equations, which tolerates CFL up to 1. This restriction is similar to that of the approach in [44] where the equation for a nonconservative field is ignored and replaced by a direct cell averaging of the dependent quantity. In terms of implementation, the evaluations are performed one cell face at a time and we accumulate their contributions to each cell depending on the signs of $S_{L}$ and $S_{R}$.

\subsection{Revisiting the contact problem}

We now revisit the example in $\S 2$ that used the original Euler equations without special treatment for the nonideal EOS shown in figure 1. The physical and numerical parameters of the problem are kept the same but we use the new generalized Roe and HLLC schemes of the previous sections. Note that the value 
of $a$ in our test problem (which is the real value for Nitrogen) is much larger than that used in the tests shown by [7]. Figure 2 shows results with the new methods for density, velocity and pressure. The velocity and pressure are shown by first subtracting the initial values (which are constants) to highlight that there are no observable small-scale fluctuations in the solution. The scale chosen in Figure 2(b-c) is just above the relative machine accuracy for these fields, about 14 digits. In addition, we can see that the density profile moved at the correct speed and matches the position of the exact solution. Furthermore, there are no observable differences between the Roe and HLLC schemes. The contact problem itself is quite simple and our experience is that when the numerics ensure the mechanical equilibrium property, little is to be observed as difference between methods. The figure also shows results using the Roe method proposed by Shyue [7]. That method uses a heuristically-derived Roe matrix that is correct for contact problems; because the non-conservative term in Eq. (22) is identically zero in such a case. As expected, the results are indistinguishable from those obtained by our proposed solvers. This will not happen when shocks are involved, as we discuss below, because the conservative-form solvers proposed in [7] are not consistent with Eq. (22) when the velocity is not constant.

\subsection{One-dimensional Riemann problem}

The second test is a Riemann problem for Nitrogen with the same density difference as before but with different pressures at both sides of the interface. The initial conditions are given by

$$
\{\rho, p\}=\left\{\begin{array}{l}
\left\{497.417 \mathrm{~kg} / \mathrm{m}^{3}(100 K), \quad 400 \mathrm{bar}\right\} \quad x<0, \\
\left\{13.33117 \mathrm{~kg} / \mathrm{m}^{3}(1000 K), \quad 40 \mathrm{bar}\right\} \quad 0 \leq x,
\end{array}\right.
$$

while the initial velocity is zero. This test was conducted on a grid that extends from -12 to $12 \mathrm{~m}$ using 1500 grid point. The CFL number was set to 0.5 to assure stability of the HLLC formulation (although one can use the Roe method with timestep twice as large). The simulation results are reported at $t=14 \mathrm{~ms}$, where the shock, contact and rarefaction have developed well and are clearly seen. The exact solution of this Riemann problem was also determined by integrating the characteristic equation for the rarefaction with a highly accurate quadrature subroutine (one cannot obtain an analytical expression for the VdW EOS).

Fig. 3 shows results of this test for density, velocity, pressure and $r$, with corresponding insets highlighting special regions of the solution. Overall, all simulations using the new methods show close agreement with the exact solution. The only simulation that shows unreasonable errors is that corresponding to the original Euler equations without special numerical treatment for the presence of a nonideal EOS. Large errors are 


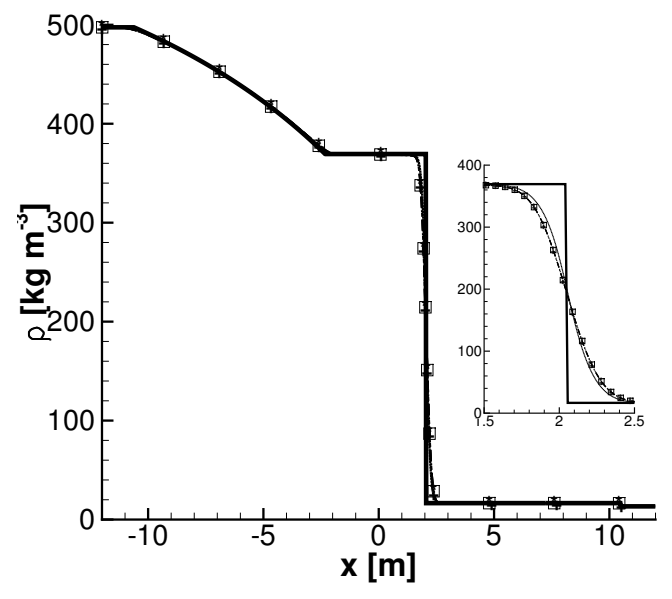

(a)

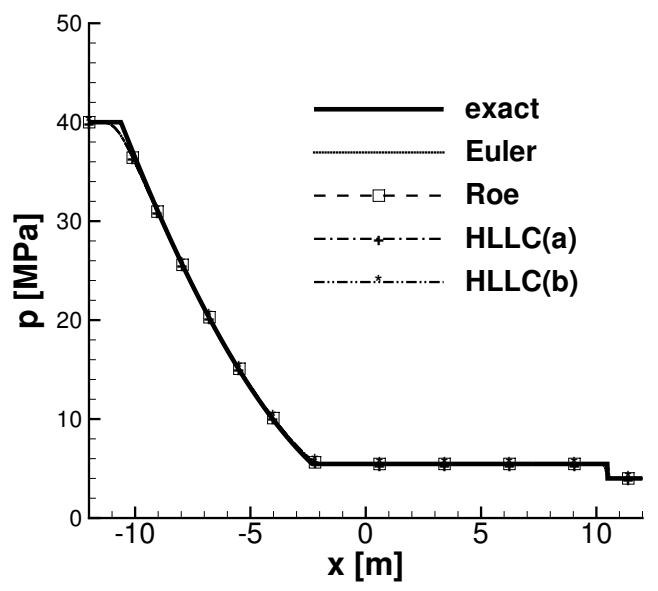

(c)

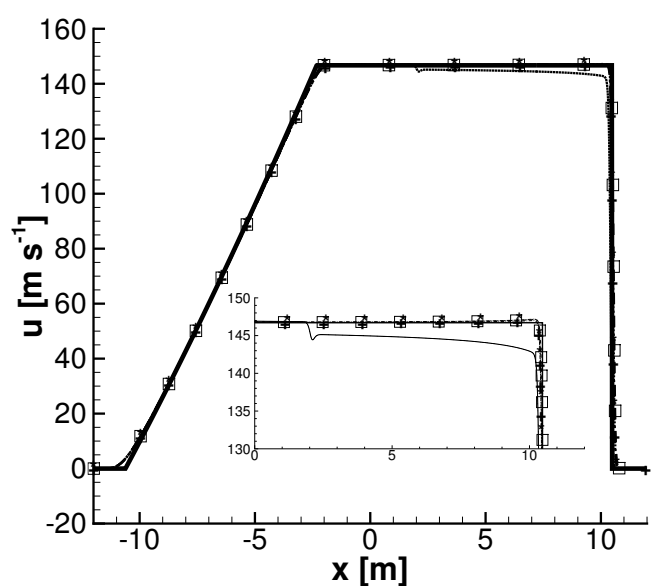

(b)

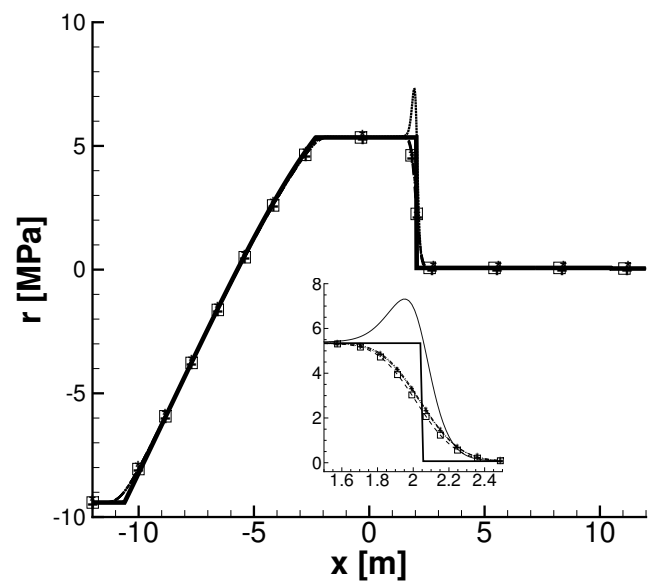

(d)

Figure 3: Density, velocity, pressure, and $r$ for the shock problem at $t=14 \mathrm{~ms}$. 

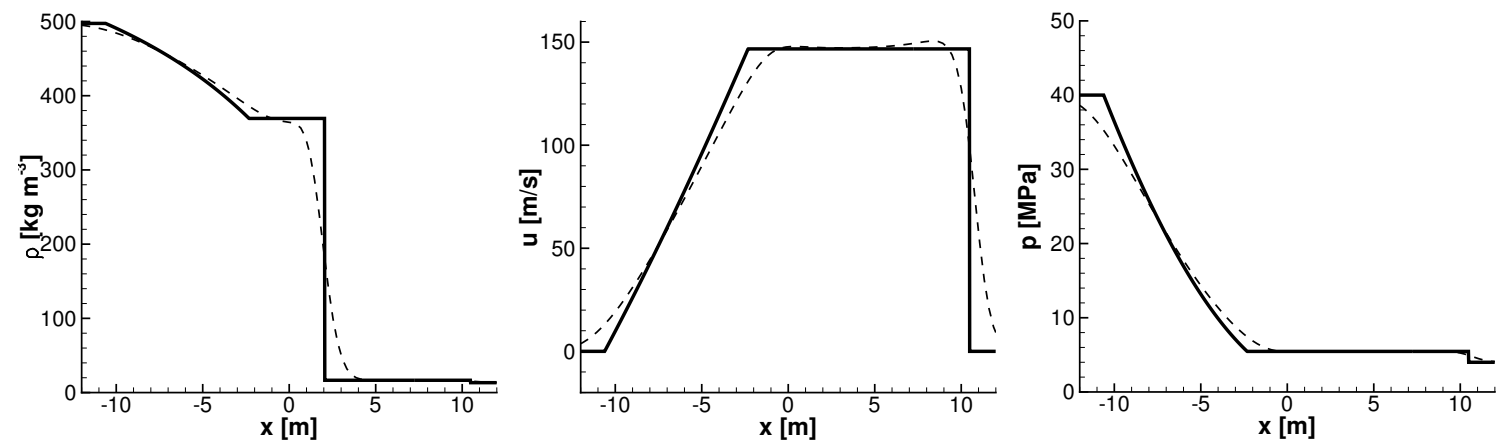

Figure 4: Coarse resolution profiles of shocktube test problem using 150 grid points at 14 ms.

present around the location of the contact, with a decrease in velocity in the right-hand side of the contact, as can be seen in the inset of figure 3(b). A more detailed comparison of $r$ obtained from the different methods and $r(\rho)$ obtained from the exact Riemann problem in the region between the contact and the shock, roughly $2<x<10$ shows relative errors of $0.2 \%, 0.035 \%$, and $0.19 \%$ for Roe, HLLC(a) and HLLC(b), respectively. As indicated in the analysis of the methods, the errors are very small but clearly HLLC(a) is the best method (in addition to being the less costly of all). For comparison purposes, we show results of simulations using HLLC(a) in figure 4 using 150 points in the same domain as before to highlight the performance of the method at coarser resolutions.

Finally, we carried out a convergence analysis and a comparison with the Roe method of Shyue [7] for this Riemann problem. Fig. 5 shows the density around the location of the shock, at $t=14 \mathrm{~ms}$, as a function of resolution using $\mathrm{N}=188,375,750,1500$ and 3000 grid points. Fig. 5(a) shows the Roe method of Shyue and Fig. 5(b) show the HLLC(a) method with the extended Euler system and our treatment of nonconservative products (equal symbols correspond to the same resolution). On one hand, it is observed that density approaches the exact solution with increasing resolution in a monotonic manner for the HLLC(a) method. Furthermore, the maximum and minimum density around the shock is very nearly bounded by the values of the exact solution. On the other hand, the Roe method of Shyue does not behave in the same manner: improvement with resolution is not as fast as with the HLLC(a) method, and it is not even clear that the method converges to the exact solution; up to the grid sizes we used. In addition, there is an overshoot of the density on the post-shock region. These problems are not completely surprising since the Roe method of Shyue was not developed from the non-conservative equations. Instead, the non-conservative term in Eq. (22) was in fact partially ignored in the definition of that Roe matrix, which was determined 


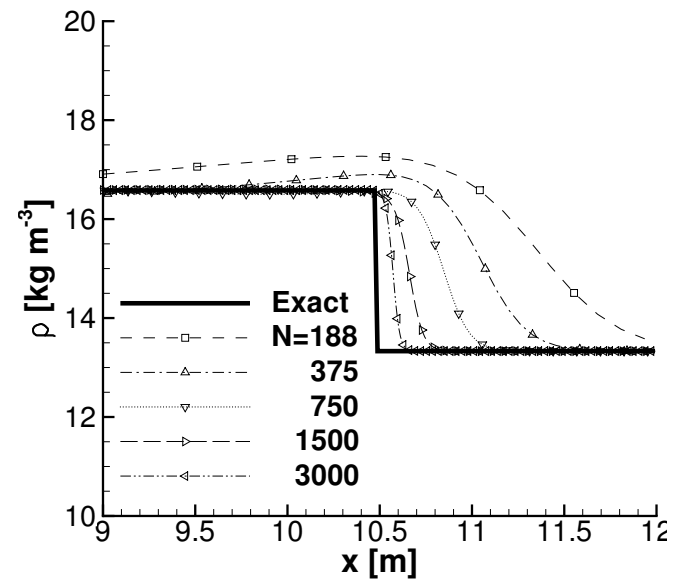

(a) Roe of Shyue

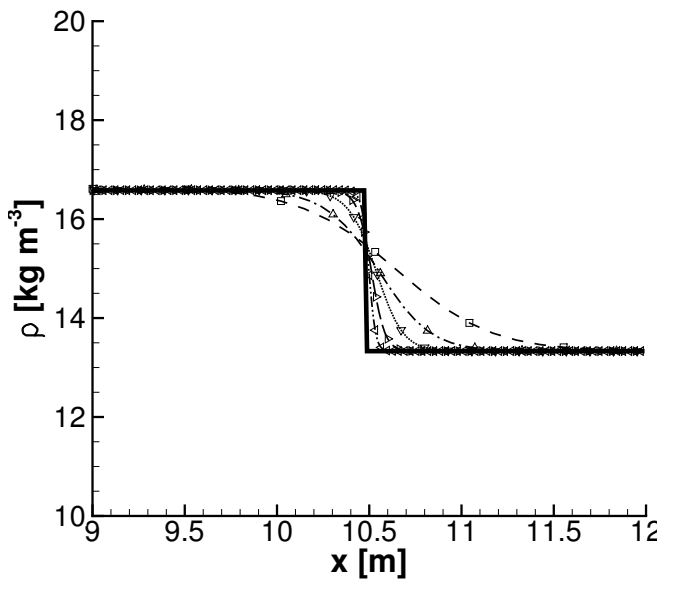

(b) $\operatorname{HLLC}(\mathrm{a})$

Figure 5: Convergence study and comparison of Roe method of Shyue (left) and HLLC(a) with extended Euler system (right).

heuristically by following the system matrix, Eq. (31), as if Eq. (6) existed (it does not). Unfortunately, the Roe matrix that mimics the system matrix is not the correct object for this problem owing to the nonconservative nature of the equations. The procedure detailed in $\S 5.1$ is one of the acceptable methodologies that ensures Eq. (32).

\subsection{One contact - one shock problem}

We consider here the one contact and one shock wave problem discussed in [9], with initial condition

$$
u_{L}=u_{R}+\sqrt{\left(p_{L}-p_{R}\right)\left(\rho_{R}^{-1}-\rho_{R}^{*-1}\right)}, \quad u_{R}=0, \quad \rho_{L}=4, \quad \rho_{R}=1, \quad \rho_{R}^{*}=2, \quad p_{R}=10^{5}
$$
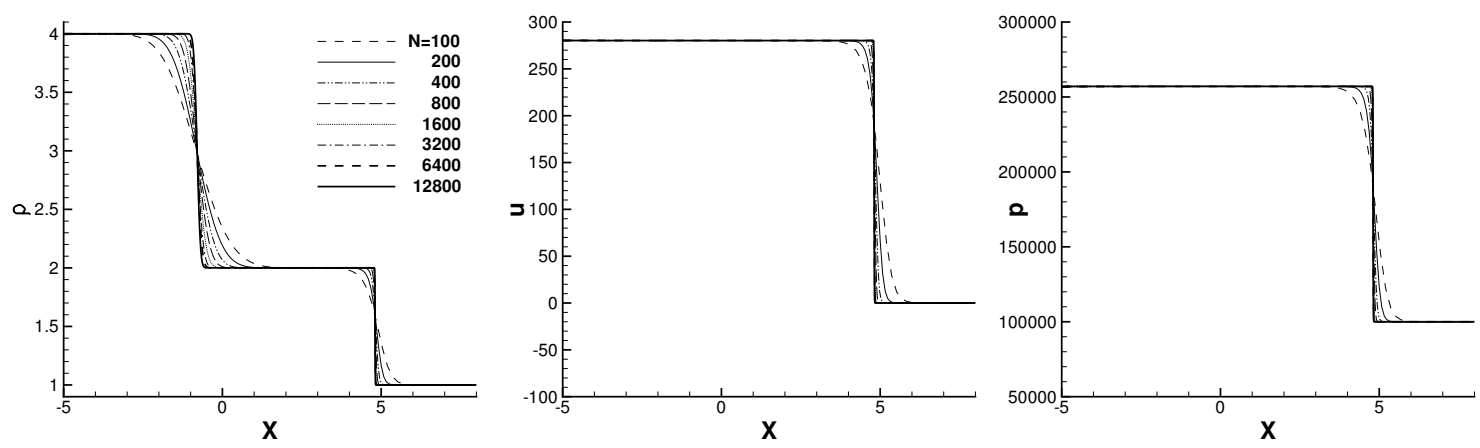

Figure 6: Density, velocity and pressure for the 3-shock wave problem using the HLLC(a) method. 


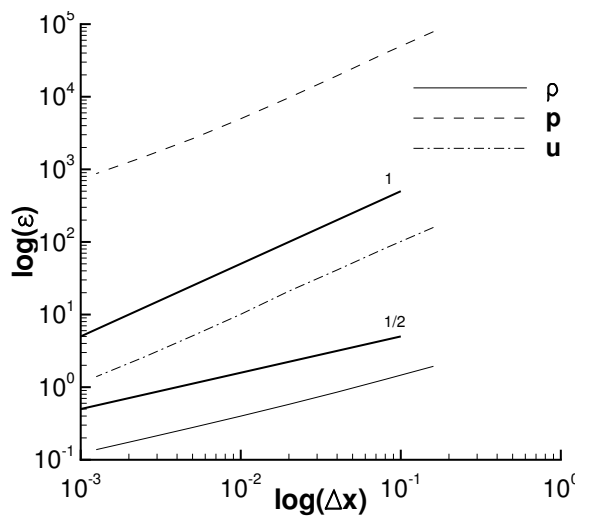

Figure 7: $L^{1}$ error of density, velocity and pressure as a function of resolution at $t=0.02$. Thick continuous lines denote reference square root and linear scalings.

and $p_{L}>p_{R}$ obtained from the solution of

$$
2 \rho_{R}^{*} \rho_{R}\left[e\left(p_{L}, \rho_{R}^{*}\right)-e\left(p_{R}, \rho_{R}\right)\right]=\left(p_{L}+p_{R}\right)\left(\rho_{R}^{*}-\rho_{R}\right)
$$

for a van der Waals gas with $a=1684.54, b=0.001692$, and $\gamma=1.329$. The intermediate states of the Riemann problem are indexed by ${ }_{L}^{*}$ and ${ }_{R}^{*}$, from left to right, respectively. The internal structure of this Riemann problem corresponds to a contact and a shock propagating towards the right; the left wave has zero strength, is a so-called "ghost" wave. Fig. 6 shows a grid convergence study for this problem with grids ranging from 100 to 12,800 points using the HLLC(a) method at $t=0.02$. The figures shows the convergence of the profiles with increasing resolution towards a solution that is expected to match the true exact solution of the problem. To verify that such exact convergence is obtained, figure 7 shows $L^{1}$ convergence as a function of grid resolution for density, velocity, and pressure. The results are consistent with the expected order of the method and with the observations of [9] (for a different method). Velocity and pressure converge linearly with $\Delta x$ while density converges as $\sqrt{\Delta x}$ owing to numerical diffusion of the contact interface; as expected. The clean convergence rate of all the fields is encouraging and suggests that the non-conservative treatment does not pollute the order of the method.

\section{The role of the path of integration}

Here, we consider further details and possible simplifications of the proposed HLLC method that can result in more computationally advantageous implementations. 


\subsection{Effects of shock jump condition}

In this section we consider a simplified version of scheme Eq. (58), in fact the simplest variant where the matrix $A$ is taken constant during the time step and computed with the cell center variables. Using this simplification, Eq. (58) becomes

$$
Q_{i}^{n+1}=Q_{i}^{n}-\frac{\Delta t}{\Delta x}\left(F_{i+1 / 2}-F_{i-1 / 2}\right)-\frac{\Delta t}{\Delta x} A_{i}^{n}\left(Q_{i+1 / 2}-Q_{i-1 / 2}\right) .
$$

Several options are possible to compute the flux of the nonconservative ' $r$ ' field:

a) The first option corresponds to that given in Eq. (61), which can also be rewritten as

$$
r_{K}^{*}=r_{K} \frac{\rho_{K}^{*}}{\rho_{K}}
$$

This intermediate state is compatible with a simplified version of Eq. (22), where ' $r$ ' obeys a conservation law-type equation of the form

$$
\frac{\partial r}{\partial t}+\frac{\partial(u r)}{\partial x}=0
$$

b) The second option assumes no jump across the right and left-facing waves

$$
r_{K}^{*}=r_{K}
$$

This intermediate state is compatible with another simplified version of Eq. (22), where ' $r$ ' obeys the transport equation

$$
\frac{\partial r}{\partial t}+u \frac{\partial r}{\partial x}=0
$$

c) The third option considers the full version of Eq. (22), that can be written as

$$
\frac{d r}{d t}=\frac{d r}{d \rho} \frac{d \rho}{d t} .
$$

An approximate intermediate state of this equation is given by

$$
r_{K}^{*}=r_{K}+\left(\frac{d r}{d \rho}\right)_{k}\left(\rho_{K}^{*}-\rho_{K}\right)
$$

d) The last option considers also the full version of Eq. (22) and uses the exact jump condition

$$
r_{K}^{*}=r\left(\rho_{K}^{*}\right)
$$

where the function $r(\rho)$ is given by Eq. (19). 
Indeed, Eq. (22) is an interesting example of nonconservative equation that admits an exact jump condition when combined with the original Euler equations. In the frame of the HLLC solver the density $\rho_{K}^{*}$ is computed with the first relation of Eq. (59).

Computed results for the same shock tube test problem as before are shown in Fig. 8. The pressure profile shows best agreement on shock position with the jump conditions in Eqs. (68)-(69) and worst results Eqs. (71)-(72). These results show that the closer the jump conditions fit the physics implied in Eq. (22) the worst are the results. This is explained by the fact that when the jump condition tends to the full physical meaning of this equation the closer the solution approaches the original Euler equations with the VdW EOS, that produces pressure oscillation. In other words, the original drawbacks are recovered if the numerics follow too closely the physics of the original Euler system. Such wrong behavior appears clearly in the profiles for the ' $r$ ' field where the same non-monotonic profile observed in the contact discontinuity example is present. The velocity profiles highlight inaccuracies of the various methods and benefits of the new one when $r_{K}^{*}=r_{K}$ or $r_{K}^{*}=r_{K} \frac{\rho_{K}^{*}}{\rho_{K}}$ jump conditions are used. The general observation is that nonlinear numerical diffusion effects in the density and $r$ field are more important than exact physical compatibility. Although $r$ diffuses independently of the density, it does it consistently with the pressure and this prevents the appearance of oscillations.

To confirm these observations we consider again the same test problem as in Fig. 2 (transport of a density discontinuity in a uniform pressure and velocity flow field) that is now shown in Fig. 9. It is first observed that the method using the exact jump conditions for ' $r$ ' produces inaccurate results for this test problem. The conventional method based on the original Euler equations with VdW EOS produces spurious oscillations as reported earlier. The graphs related to the ' $r$ ' field show the non-monotonic behavior of this method at contact waves while it becomes monotonic with the present method. These results show that the jump conditions of the nonconservative equation are not the key point of accurate computations in flows of supercritical fluids. As explained formerly, accuracy is more related to the numerical diffusion of density and not to shock compression. To check once more this observation a test case involving very strong shocks is considered in the next section in conjunction with additional considerations.

\subsection{Comparison with a nonconservative pressure equation formulation}

The key point of the present paper relies on the nonconservative Eq. (22) that implies a pressure balance compatible with the nonlinear evolution of density. As it is a pressure equation, an option could be to consider the full pressure equation. Such an option has been investigated by various authors, see [3] for 

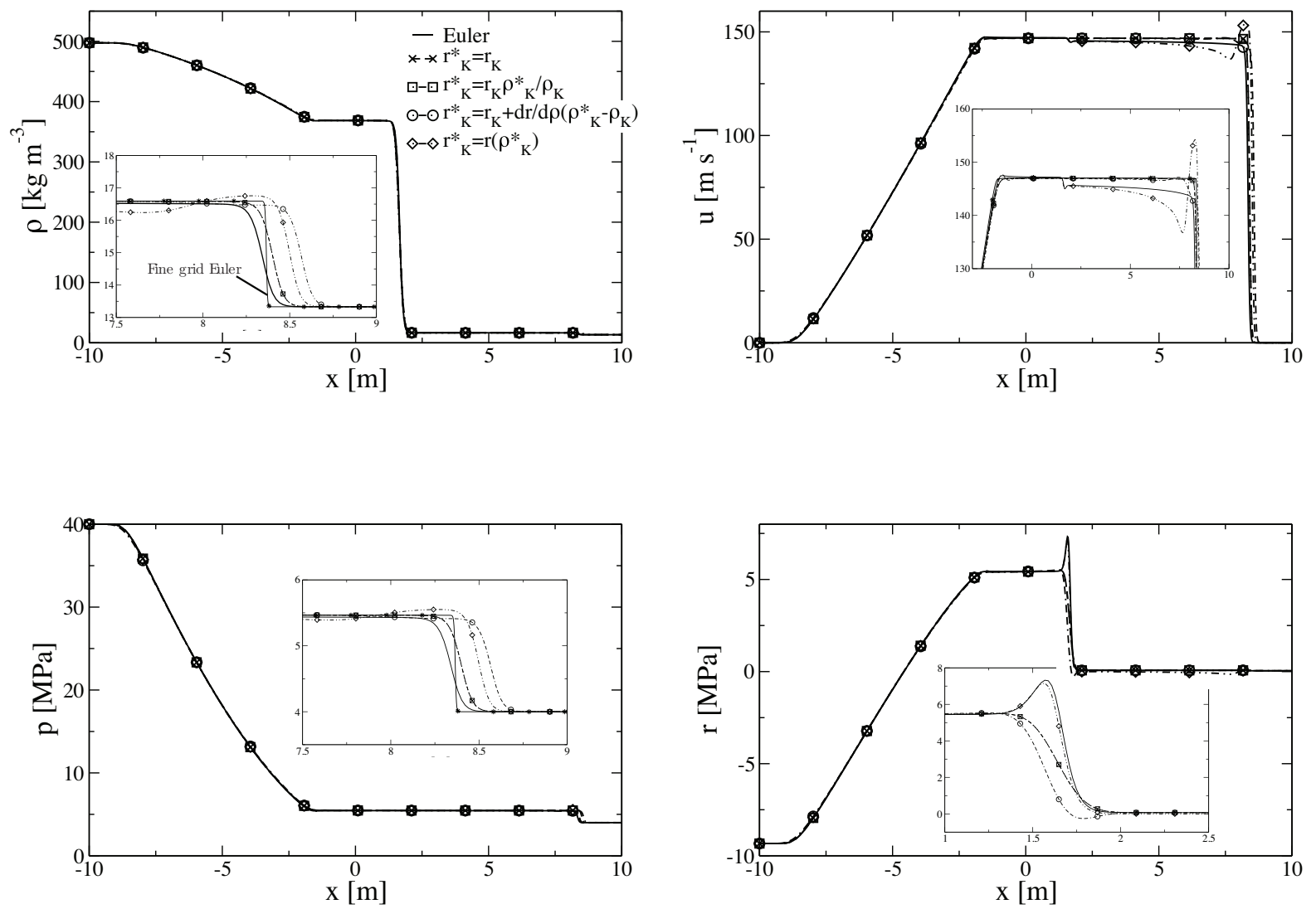

Figure 8: Shock tube test problem with supercritical states at $t=11.2 \mathrm{~ms}$. Comparison of various treatments of the nonconservative equation in the Riemann solver. The options $r_{K}^{*}=r_{K} \frac{\rho_{K}^{*}}{\rho_{K}}$ and $r_{K}^{*}=r_{K}$ provide accurate results while considering part of the physics only of the full equation (22). The options $r_{K}^{*}=r_{K}+\left(\frac{d r}{d \rho}\right)_{k}\left(\rho_{K}^{*}-\rho_{K}\right)$ and $r_{K}^{*}=r\left(\rho_{K}^{*}\right)$ fit better the physics of this equation but produce wrong results. 

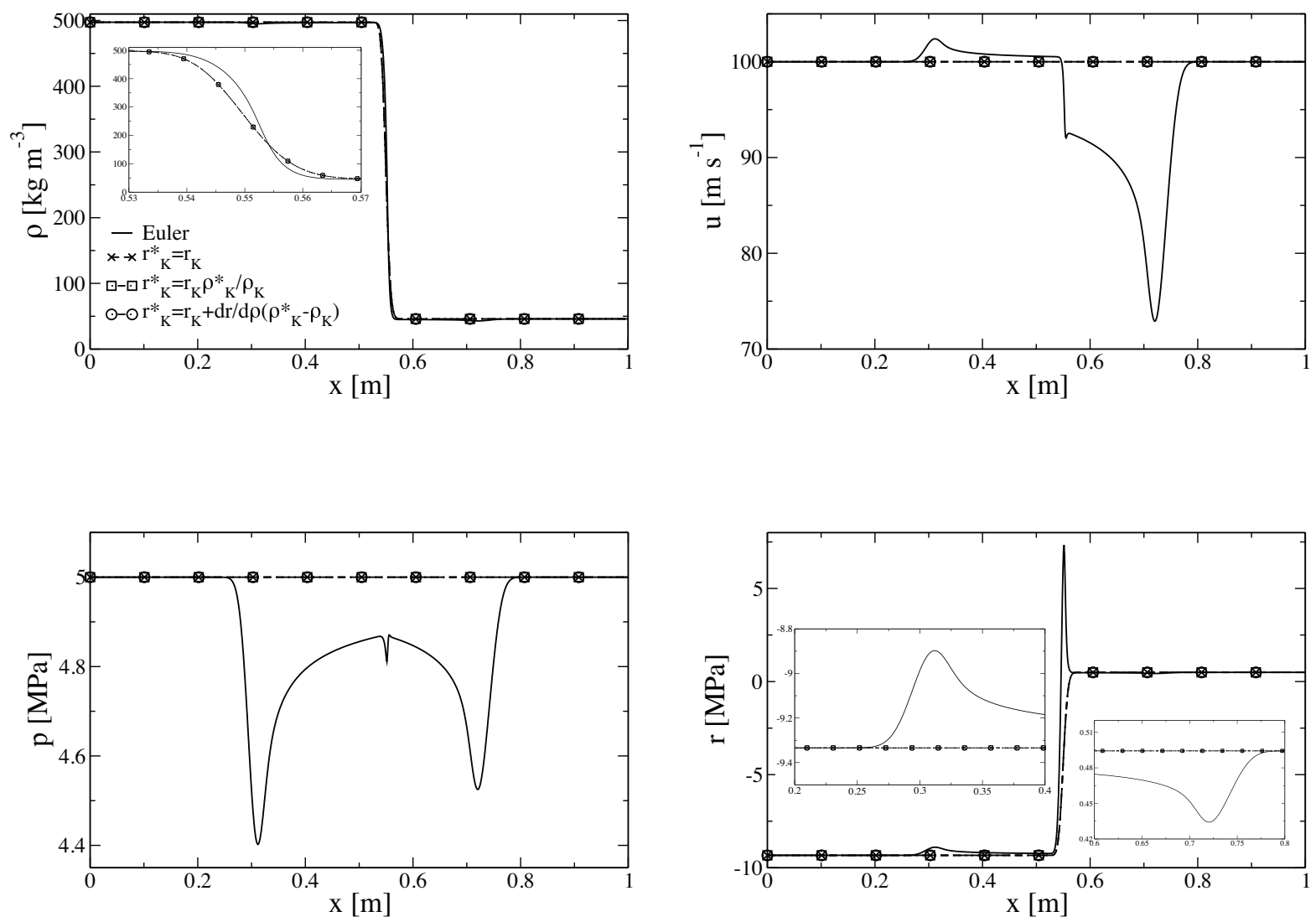

Figure 9: Transport of a density discontinuity in a uniform pressure and velocity flow field at $t=0.5$ ms. Comparison of various treatments of the nonconservative equation in the Riemann solver. Accurate computations are obtained with $r_{K}^{*}=r_{K} \frac{\rho_{K}^{*}}{\rho_{K}}$ and $r_{K}^{*}=r_{K}$. Computational failure is observed with $r_{K}^{*}=r\left(\rho_{K}^{*}\right)$ that corresponds to the exact jump condition and results of poor accuracy are obtained with $r_{K}^{*}=r_{K}+\left(\frac{d r}{d \rho}\right)_{k}\left(\rho_{K}^{*}-\rho_{K}\right)$. 
example. The pressure equation reads,

$$
\frac{\partial p}{\partial t}+\frac{\partial(p u)}{\partial x}+\left(\rho c^{2}(p, \rho)-p\right) \frac{\partial u}{\partial x}=0
$$

This equation having exactly the same structure as the equation for ${ }^{6}$ ', Eq. (22), allows us to use the same algorithm as before with analogous intermediate state $\left(p_{K}^{*}=p_{K} \frac{\rho_{K}^{*}}{\rho_{K}}\right)$ used in the computations that follow. It is worth to mention that the intermediate state related to the advection type form $\left(p_{K}^{*}=p_{K}\right)$ provides results of good accuracy as well, quite indistinguishable with the ones reported on the next figure. Also note that when the pressure equation is solved, the balance energy equation is obviously omitted and energy conservation is lost. The mass and momentum equation are still solved in conservative form.

Two shock tube computations are addressed. The first one uses the same initial data as previously and the second one considers a very strong shock. For the first of these two computations the same initial data as Fig. 8 are considered and results obtained by the original Euler equations with VdW EOS are used as reference and compared with the method promoted in the present paper, using $\left(r_{K}^{*}=r_{K} \frac{\rho_{K}^{*}}{\rho_{K}}\right)$ as jump condition in the Riemann solver for the equation for ' $r$ ' and similar formula for the equation for the pressure. The results are shown in Fig. 10. On this test case, the pressure-based formulation provides accurate results, improving those of the conservative formulation using the original Euler system. Very good accuracy is still obtained with the augmented system. To illustrate the limits of the pressure based formulation, another shock tube test case involving very strong shock is considered in the Fig. 11. As expected and consistent with well known behavior, the pressure formulation fails and provides wrong shock speed as well as wrong pressure and density jumps. Adaptive primitive-conservative variable methods could be used to alleviate this difficulty [10].

In general, demonstrating convergence of solutions to non-conservative equations is no trivial matter. Abgrall \& Karni [42] discussed some of the difficulties that one faces even with apparently simple problems, where solutions of a non-conservation system derived from a conservative system (Euler in this case) does not converge to correct viscosity solution of the original problem. One difficulty that appears to be shared by these systems is that the unavoidable numerical viscosity of any shock-capturing method tends to interfere with the non-conservative product treatment. For low-order methods, the amount of numerical viscosity may overwhelm the effect of path of integration and result in non-converging results [45]. It appears that much more careful investigation is required, likely using high-order methods, to demonstrate convergence of discontinuous solution to general non-conservation form equations. These difficulties do not seem to be a problem for the methods discussed in this communication since we have shown convergence in multiple 

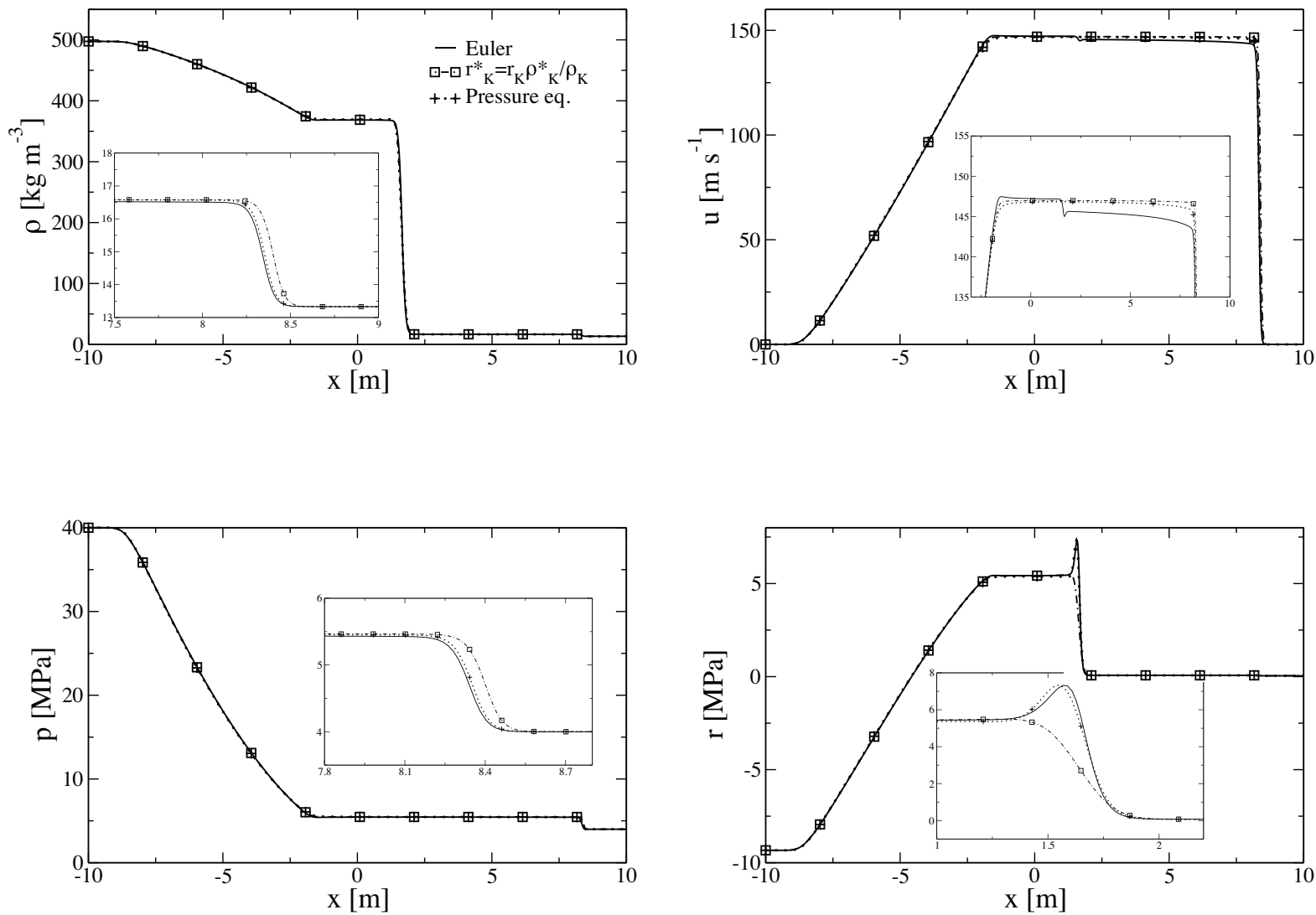

Figure 10: Shock tube test problem with supercritical states at $t=11.2 \mathrm{~ms}$ considering same initial states as Fig. 8 . Comparison of various formulations: conservative with original Euler equations and VdW EOS, nonconservative with the equation for ' $r$ ', Eq. (22), and nonconservative with the pressure equation. Accurate results are obtained with the two nonconservative formulations. 

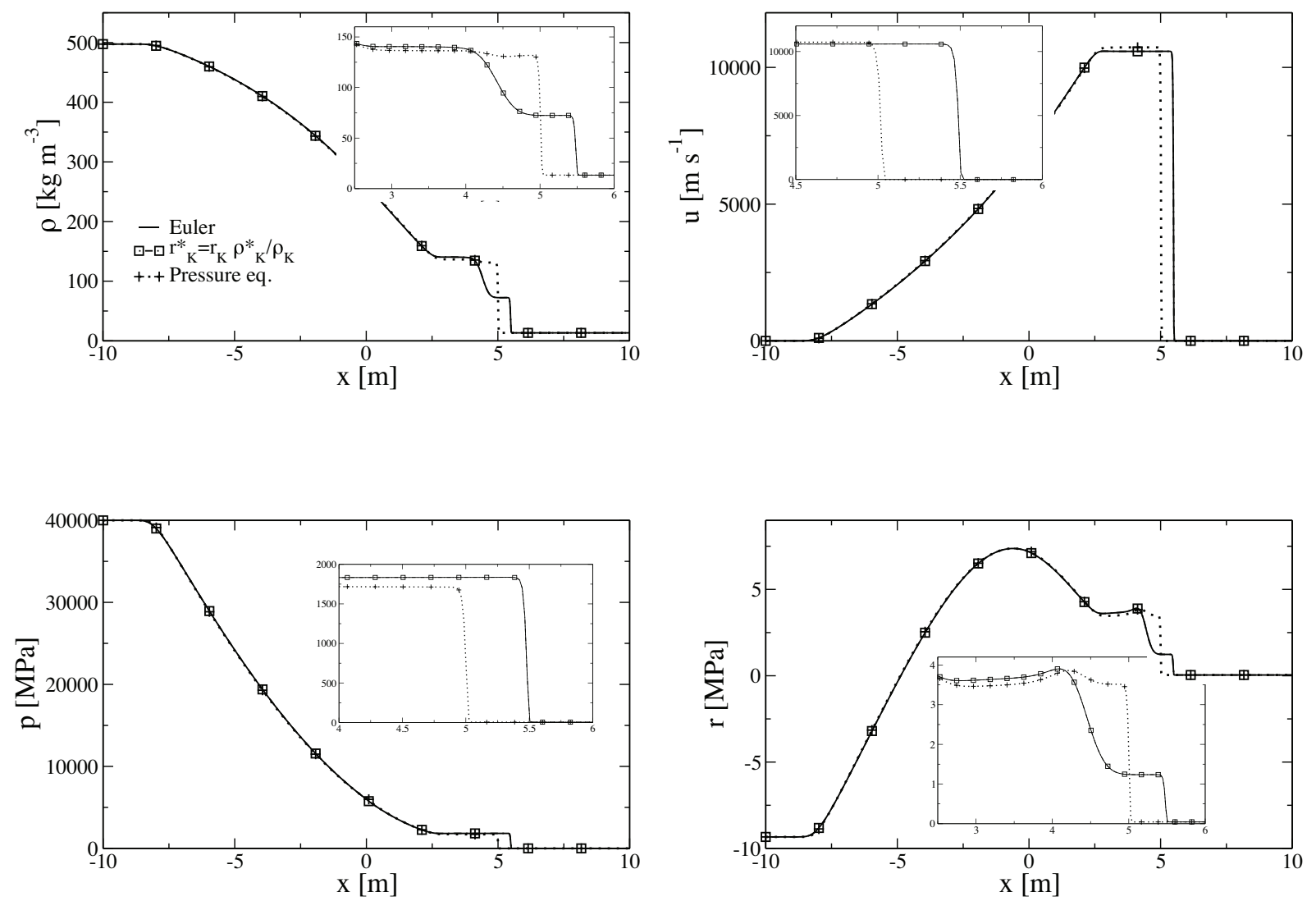

Figure 11: Shock tube test problem with supercritical states and extreme initial data resulting in very strong shock at $t=$ $0.42 \mathrm{~ms}$. Comparison of various formulations: conservative with original Euler system, nonconservative with Eq. (22) and nonconservative with the pressure equation. Now the pressure based formulation shows its limits with wrong shock speed while the one based on the equation for ' $r$ ' remains accurate. 
examples without any special care and using the first-order accurate methods we discussed.

\subsection{Temperature calculation}

In this section, the temperature field obtained from the various studied formulations (original Euler equations, nonconservative with the equation for ' $r$ ', Eq. (22), and nonconservative with the pressure equation) is examined for the transport of the contact discontinuity and the shock tube problem. Using the original

Euler equation together with the $\mathrm{VdW}$ EOS, the temperature is uniquely determined from the density and internal energy as follows

$$
T=\frac{(\gamma-1)\left(\rho e+a \rho^{2}\right)}{R \rho} .
$$

The situation differs when Eq. (22) is used. Among other possible expressions, the temperature can be evaluated directly using Eq. (73) as before or from the pressure and density, according to

$$
T=\frac{(1-\rho b)\left(p+a \rho^{2}\right)}{R \rho} .
$$

A comparison between these three methods is offered in Fig. 12 (left) for the transport of a contact discontinuity. The temperature field is no longer monotonic if Eq. (73) is used. The convex state of the EOS is recovered when temperature is computed from Eq. (74). The latter seems to be a proper choice for the determination of temperature when using the additional ' $r$ ' field. This is confirmed in Fig. 12 (right) for the shock tube calculation, where the temperature obtained using Eq. (74) is virtually equal to that obtained using the original Euler equations on a fine grid.

\section{Treatment of slip lines}

The generation of spurious oscillations or lack of mechanical equilibrium is not exclusive to the presence of a contact surface in a gas with complex EOS. It can also arise owing to the presence of a slip line (plane) when the tangential velocity is discontinuous. A numerical strategy to address this problem was suggested by [14] and further developed by [7]. The idea is to start from the equation for $\rho E$, i.e.,

$$
\frac{\partial \rho E}{\partial t}+u_{o} \frac{\partial \rho E}{\partial x}=0,
$$

and observe that in the presence of a jump in the tangential velocity, Eq. (14) becomes

$$
\rho E=q p+r+\mathcal{K},
$$



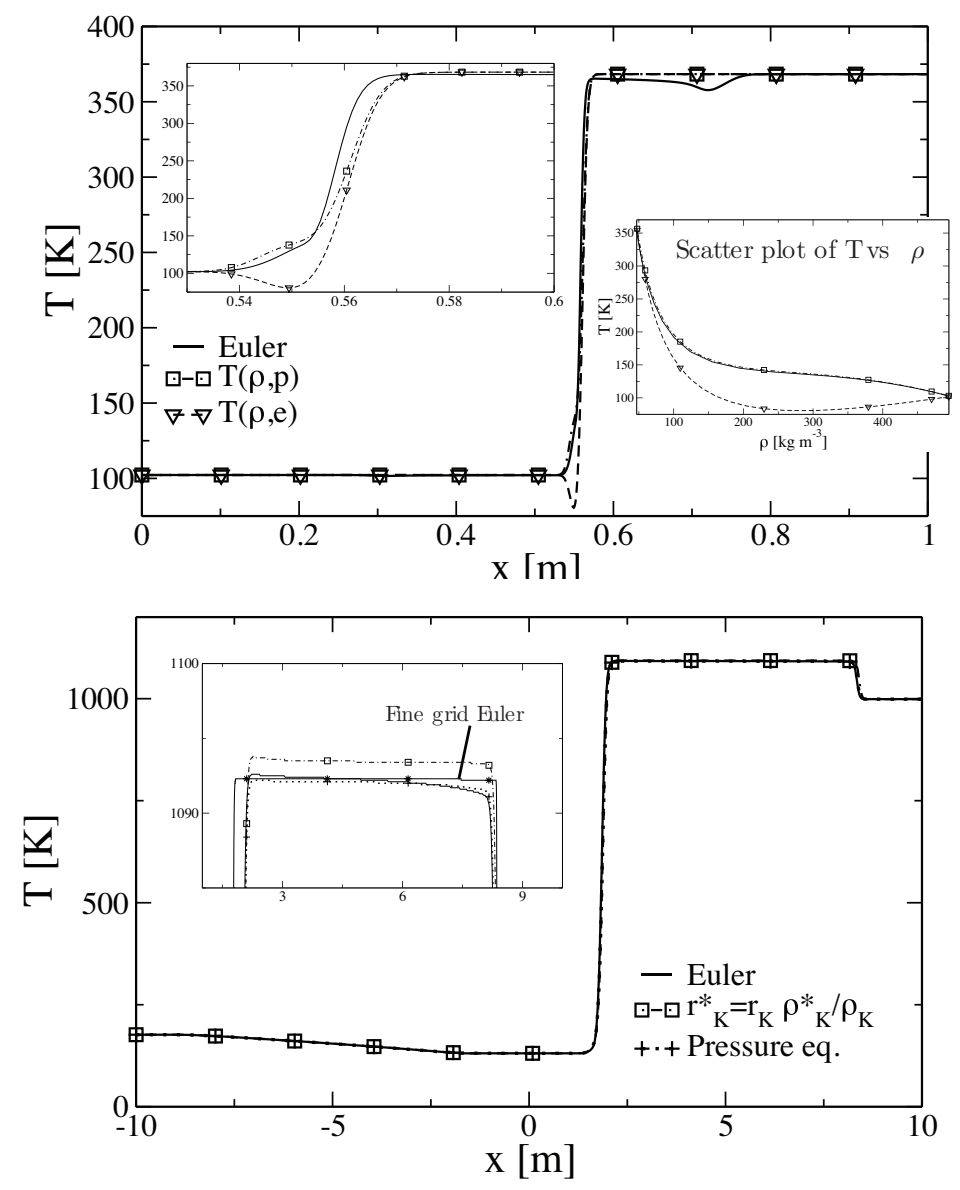

Figure 12: Temperature for (left) the transport of a density discontinuity in a uniform pressure and velocity flow field at $t=0.5$ $\mathrm{ms}$ and (right) the shock tube test problem with supercritical states at $t=11.2 \mathrm{~ms}$. 
where the kinetic energy of the fluid per unit volume is defined as

$$
\mathcal{K}=\frac{1}{2} \rho\left(u^{2}+v^{2}\right)
$$

with $u$ and $v$ denoting the normal and tangential velocity components, respectively. Now, restoring exact mechanical equilibrium requires that $\mathcal{K}$ obeys an equation of the form Eq. (75), which for general $(u, v)$ it can be shown to be

$$
\frac{\partial \mathcal{K}}{\partial t}+\frac{\partial(u \mathcal{K})}{\partial x}+\frac{\partial(v \mathcal{K})}{\partial y}+u \frac{\partial p}{\partial x}+v \frac{\partial p}{\partial y}=0
$$

The inclusion of Eq. (78) as part of the extended system, in addition to the equation for 'r', can be implemented without structural modifications to the HLLC methods we discussed previously, simply by replacing Eq. (49) by

$$
c_{o}^{2}=\tilde{\Gamma}\left(\hat{H}-2 \hat{\omega}-\hat{\mathcal{K}}+\hat{\alpha}+\kappa \tilde{\alpha}_{1}\right),
$$

where

$$
\hat{\mathcal{K}}=\frac{\mathcal{K}_{L} / \sqrt{\rho_{L}}+\mathcal{K}_{R} / \sqrt{\rho_{R}}}{\sqrt{\rho_{L}}+\sqrt{\rho_{R}}} .
$$

This doubly-extended system of equations is referred-to as the 'rs' Euler equations later on. An important modification that this additional extension requires is a treatment of the non-conservative product (last two terms) in Eq. (78). This can be accomplished by following the procedure explained in Eq. (65), which now becomes the integral (for the first term and analogously for the second term)

$$
\begin{aligned}
\int_{0}^{1} u \frac{d p}{d s} d s & =\int_{0}^{1} \frac{w_{2}(s)}{w_{1}(s)} \frac{d p}{d s} d s \\
& =\left(\int_{0}^{1} \frac{w_{2}(s)}{w_{1}(s)} \frac{d Z^{*}}{d w_{1}}\left[w_{3}(s)-w_{4}(s)-w_{6}(s)\right] d s\right) \Delta w_{1}+\left(\int_{0}^{1} \frac{w_{2}(s)}{w_{1}(s)} Z^{*}(s) d s\right)\left(\Delta w_{3}-\Delta w_{4}-\Delta w_{6}\right),
\end{aligned}
$$

where

$$
W=\left(w_{1}, w_{2}, w_{5}, w_{3}, w_{4}, w_{6}\right)=\{\sqrt{\rho}, \sqrt{\rho} u, \sqrt{\rho} v, \sqrt{\rho} H, r / \sqrt{\rho}, \mathcal{K} / \sqrt{\rho}\}
$$

and the pressure expressed in terms of these variables becomes simply

$$
p=Z^{*}\left[w_{3}-w_{4}-w_{6}\right], \quad \text { where } Z^{*}=\frac{(\gamma-1) w_{1}}{\gamma-b w_{1}^{2}},
$$

(we have not renamed the $w_{k}$ variables to avoid confusion with the one-dimensional formulation).

As noted by [7], more work is required to modify the Roe method because of the coupling between eigenvectors of the generalized Roe matrix. Therefore, it is preferable to concentrate on the HLLC methods we presented before given that the Roe method is computationally more expensive. To this end, figure 13 


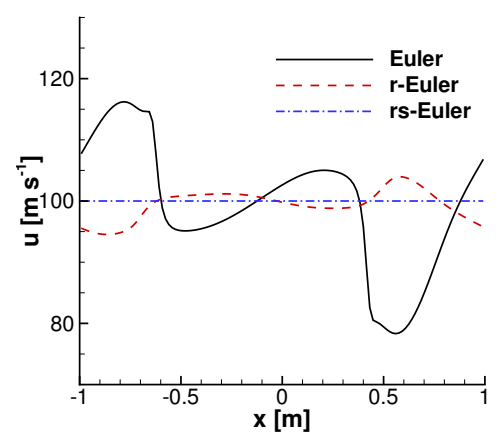

(a)

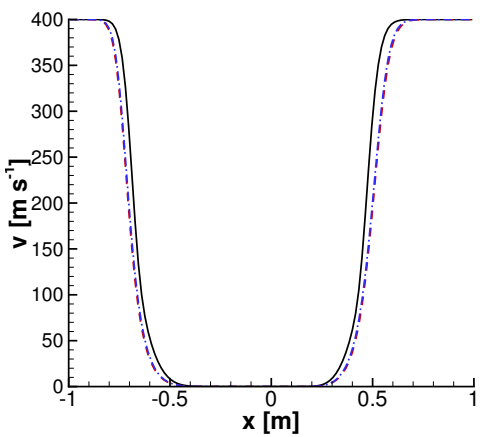

(b)

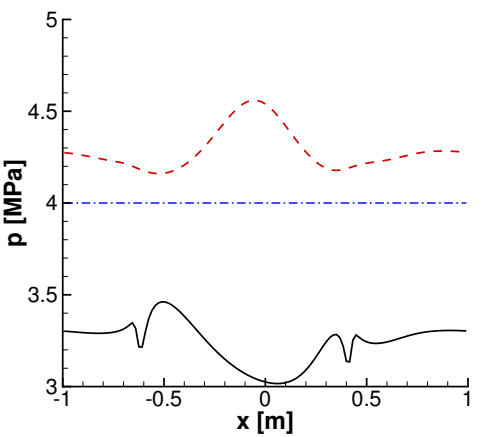

(c)

Figure 13: Comparison of the original, extended 'r' only and slip extended 'rs' Euler systems using the HLLC(a) method. From left to right: $u$ and $v$ velocity, and pressure at $t=4 \mathrm{~ms}$.

shows comparisons of the simple contact problem discussed in figure 2 with the addition of a slip velocity; a jump in the tangential velocity from 0 to $400 \mathrm{~m} / \mathrm{s}$ at the contact location. Since all present HLLC methods give similar results, we present profiles obtained with the best method we tested, i.e., HLLC(a). For comparison purposes, we reproduce the results of this slip-contact problem using the original conservative Euler equations, the extended system with the transport equation for ' $r$ ' and the slip-extended method, 'rs', that includes Eq. (78). Evidently the slip-extended method ensures mechanical equilibrium and therefore produces no pressure oscillations. Note that there is only minor need to modify the HLLC speed estimates according to Eq. (79).

\section{Two-dimensional formulation}

From the previous analysis we conclude that the best formulation is based on Eq. (22) and EOS given in Eq. (23), as it is able to compute accurately transport of density discontinuity and shocks of arbitrary strength. Appendix B describes the generalization required to treat tangential momentum within the present framework. We describe next two examples of injection of a column of gas into a chamber of different density. In the first case the flow velocity is uniform while in the second case the configuration corresponds properly to a jet. Finally, we present a more difficult two-dimensional Riemann problem exercising the slip-line problems discussed in the previous section.

\subsection{Injection at uniform velocity}




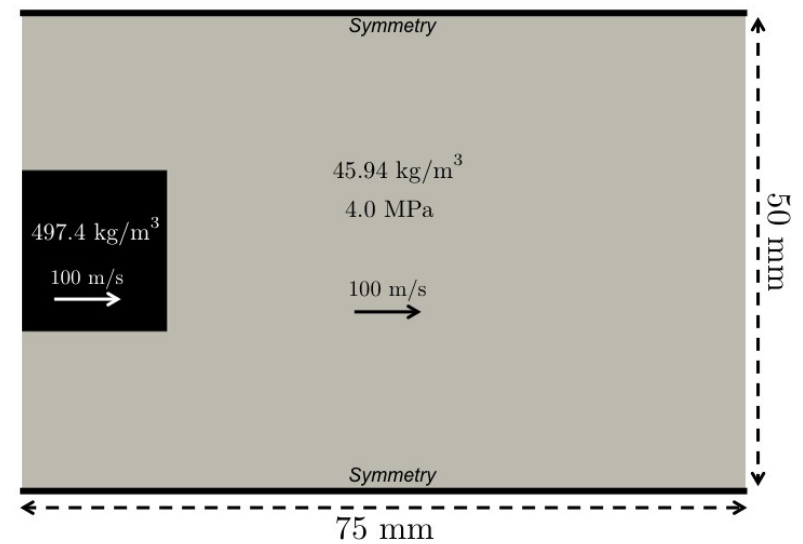

Figure 14: Sketch of simple injection problem.

The configuration examined is a simplified form of fuel injection in systems used for cryotechnic space launcher engines and the initial condition is shown in Fig. 14. Here, a heavy supercritical fluid is injected in a chamber with the same fluid at lower density. In conventional situations, the two fluids have different velocities and the created shear induces breakup of the heavy fluid jet followed by turbulent mixing. In the present configuration the fluids are assumed to have the same velocity and same pressure and simple transport of density fields should be observed. We present first the results using a Cartesian solver using direction-by-direction vector splitting. The mesh size was 300 by 200 grid points in the $\mathrm{x}$ and y directions, respectively, and comparisons are made between the original and the extended Euler system. Fig. 15 shows pressure, horizontal and vertical velocity at $90 \mu \mathrm{s}$. It is clearly observed that the proposed method is able to advect the column of high density fluid without generation of any kind of mechanical oscillations (velocity or pressure).

The method given by Eq. (67) has also been extended to two dimensions in the unstructured code DALPHADT based on a simplicial (triangular) mesh, which advances the vector of state following

$$
Q_{i}^{n+1}=Q_{i}^{n}-\frac{\Delta t}{V_{i}} \sum_{j=1}^{3} S_{j} F_{j} \cdot n_{j}-\frac{\Delta t}{V_{i}} A_{i}^{n} \sum_{j=1}^{3} S_{j} Q_{j} \cdot n_{j},
$$

where $V_{i}$ denotes the volume of the grid cell $i, S_{j}$ the area of the cell face $j=1,2,3$ enclosing cell $i, F_{j}$ the flux at that face, $A_{i}^{n}$ the chosen nonconservative matrix averaged about the grid cell and time considered. This scheme is an extension of the method detailed in [46], which can also be applied in three dimensions by extending the sums in Eq. (84) to all the faces of tetrahedral cells. 

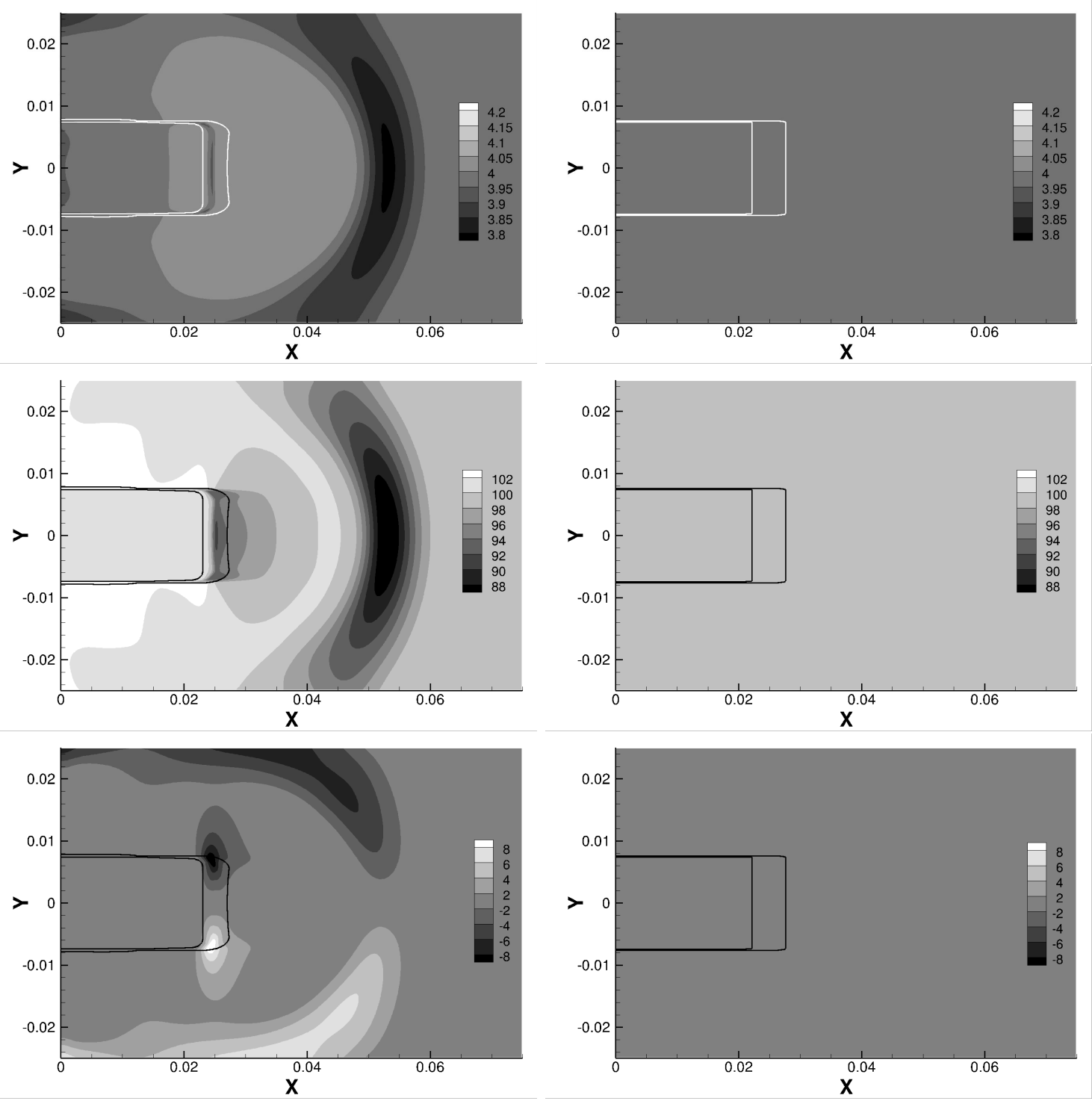

Figure 15: Simplified supercritical fluid injection test in 2D. Pressure, horizontal and vertical velocity contours (top to bottom, respectively) for the simplified injection test of Fig. 14 are shown at time $90 \mu \mathrm{s}$. Contours marked with lines indicate the inner and outer density of $\rho=50$ and $450 \mathrm{~kg} / \mathrm{m}^{3}$, respectively; (pressure is given in MPa and velocities in m/s).The graphs on the left column correspond to computations done with the Euler equations and show spurious pressure and velocity waves. Computation shown on the right column are done with the present model and are free of oscillations. 

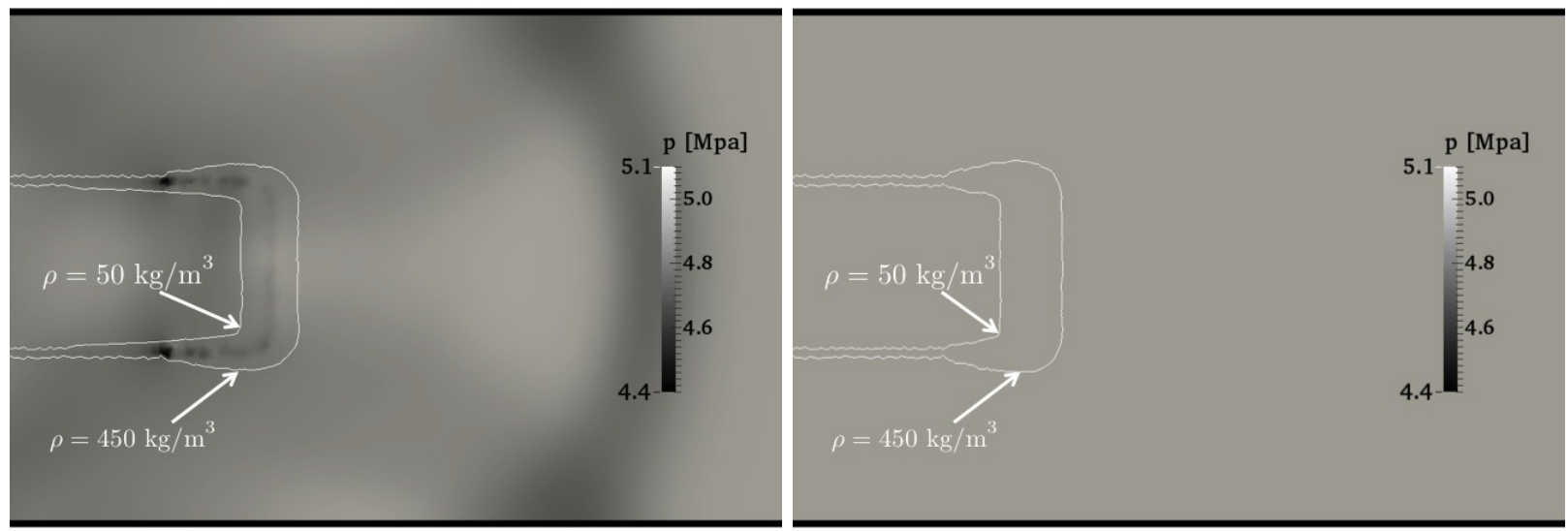

Figure 16: Pressure contours for the simplified injection test of Fig. 14 are shown at time $90 \mu \mathrm{s}$. The new method (right picture) is free of pressure oscillations while the conventional one (left picture) presents severe oscillations as well as spurious pressure waves.

Fig. 16 shows simulation results for the pressure and density fields at $90 \mu \mathrm{s}$. The results on the left figure were obtained using the original Euler equations and exhibit large pressure oscillations, while the right figure shows results obtained with the present extended model using HLLC and intermediate state given by Eq. (68) where no pressure disturbances are observed. In both cases, the contact interface is diffused predominantly in the streamwise direction while little numerical diffusion is observed along the transverse direction. This is consistent with the nature of shock-capturing methods. Importantly, the difficulties with the original Euler system for this nonideal EOS is not limited to mechanical nonequilibrium effects near or in close proximity of the contact interface (density variation region). We can also observe large pressure (acoustic like) waves propagating throughout the domain, both interacting along the transverse direction as well as propagating towards the right-hand side of the figure, far away from the contact region itself. It is common to encounter pressure variations of about fifty percent of the initial pressure in conventional simulations of this type using the original Euler system. Evidently, the results on the right do not suffer from these problems and a quiet undisturbed flow is maintained at the nominal velocity and pressure set by the initial conditions of the problem. Comparison between the Cartesian and the unstructured solver solutions highlight that the only difference between the two methods is the somewhat larger numerical diffusivity experienced by the density interface in the latter formulation, as it is expected for an unstructured mesh, while mechanical equilibrium is maintained in both cases. 


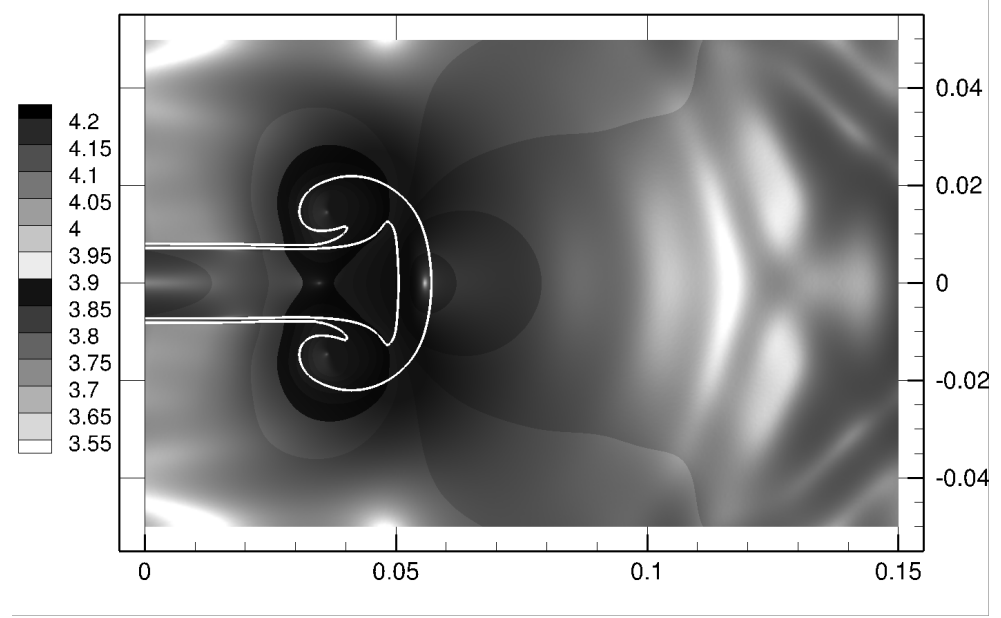

Figure 17: Pressure at time $500 \mu \mathrm{s}$ in supercritical fluid injection test with initial condition as in figure 14 but with fluid in the chamber at rest. Contours marked with lines indicate the inner and outer density of $\rho=50$ and $450 \mathrm{~kg} / \mathrm{m}^{3}$, respectively; pressure is given in $\mathrm{MPa}$.

\subsection{Jet injection}

The second two-dimensional example corresponds to a jet matching the conditions of the previous example, but where the chamber fluid is at rest. We use the Cartesian solver in this simulation. The domain size and number of grid points has been doubled to accommodate for a larger simulation time of $500 \mu \mathrm{s}$. This allows us to observe clearly the formation of the pair of vortices at the front of the high-density column. Fig. 17 shows the pressure at the end of the simulation time with the two isolines of density shown in the previous example. Note that the pressure and velocity will not be uniform in this case since the high-speed column of high-density fluid will induce a compression wave in the fluid of the chamber that is at rest. This type of simulation is more difficult to analyze qualitatively and quantitatively because there is no simple reference state to compare to. Instead, we have chosen to take selected cuts through the domain at locations that we suspected of presenting computational difficulties. The first cut is the center line of the domain, $y=0$, while the second and third cuts are across the jet column, at $x=12.4 \mathrm{~mm}$ (roughly one third along the way through the jet) and $x=40 \mathrm{~mm}$ (cutting through the vortex centers).

Fig. 18 shows these profiles for pressure and both components of velocity for the original, the ' $r$ ' and the 'rs' extended Euler systems. Careful inspection of these profiles reveals that there are multiple locations where 'glitches' in the numerical solution are evident. For example, the pressure along the centerline $(y=0)$ 

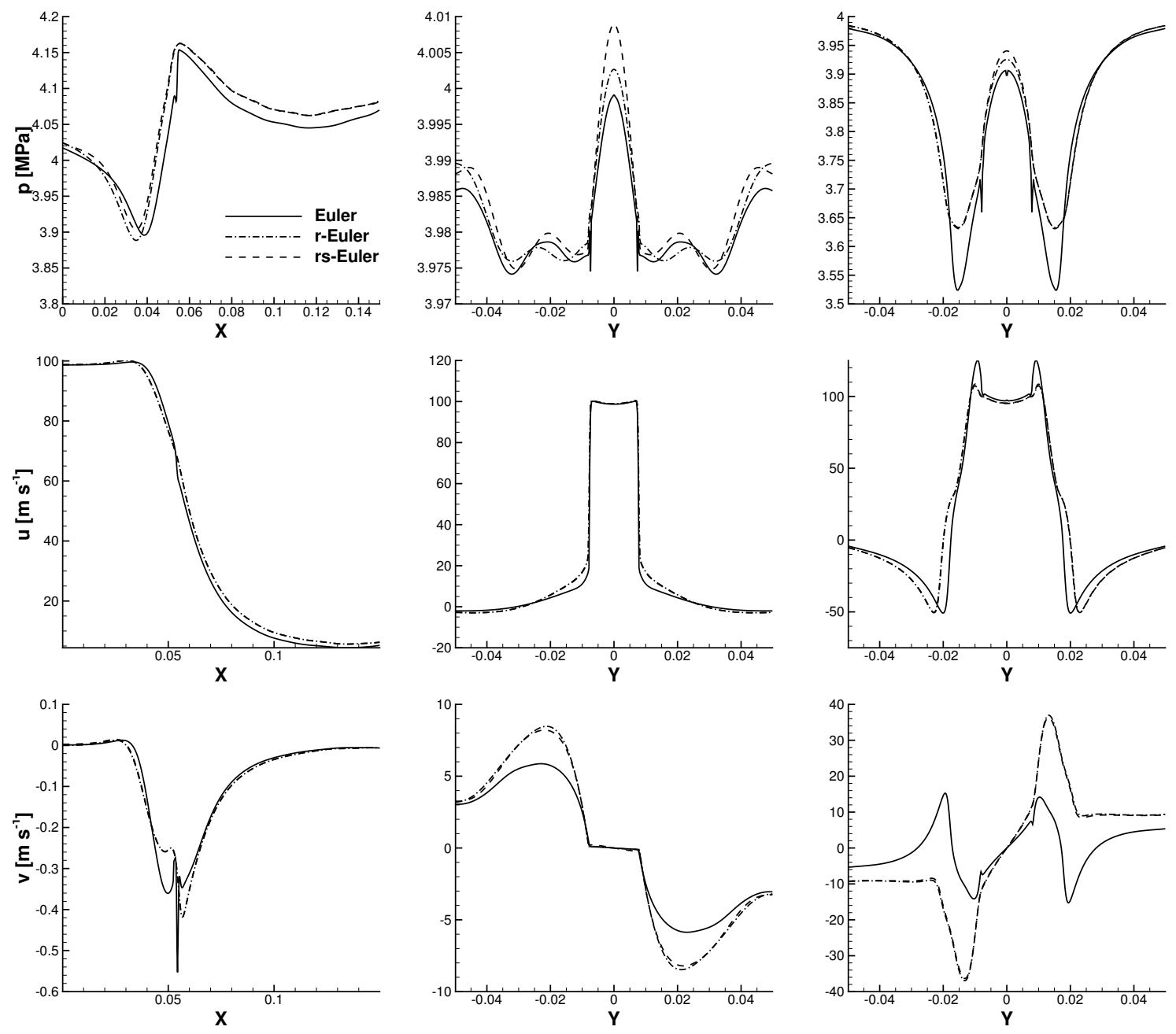

(a) $y=0 \mathrm{~mm}$

(b) $x=12.4 \mathrm{~mm}$

(c) $x=40 \mathrm{~mm}$

Figure 18: Comparison of profile cuts at time $500 \mu \mathrm{s}$ at $y=0, x=12.4$ and $x=40 \mathrm{~mm}$. From top to bottom: pressure, $\mathrm{u}$ velocity and $\mathrm{v}$ velocity. 
at around $x=60 \mathrm{~mm}$ and at the vertical cuts just at the edge of the jet show abnormal behaviors for the original Euler system, while the ' $r$ ' and 'rs' extended systems appear reasonably smooth and well behaved. Furthermore, one observes overshoots of horizontal $u$ velocity at the edge of the jet in the second vertical cut $x=40 \mathrm{~mm}$ and excessively large gradients (oscillation) of vertical $v$ velocity at the head and edge of the jet along the second vertical cut. The improvements of the extended system are encouraging and suggestive of better behavior than that of the original Euler system. There are very small differences between the ' $r$ ' and 'rs' extended system, which in the figure are only noticeable for the pressure at the $x=12.4 \mathrm{~mm}$ cut. We will explore the benefits of the 'rs' extended system in the next example.

These results highlight that the present method can have beneficial properties in more complex LES and DNS simulations where three-dimensionality, time-dependent effects, turbulence and other physically important details are present and one would not like to pollute these fields by extraneous numerical artifacts.

\subsection{Two-dimensional Riemann problem}

The previous examples are illustrative of injection problems encountered in propulsion where the nonlinearity of the EOS can be problematic. Here we consider another class of problems produced by the presence of strong slip lines; effectively two-dimensional Riemann problems. This problem is an adaptation of that used in [7] where we have essentially modified the reference thermodynamic state to be closer to the critical point of the fluid, where the nonlinearity of the EOS is stronger. The initial conditions are given by

$$
\begin{aligned}
& (\rho, u, v, p)_{1}=\left(\rho_{0}, u_{0},-v_{0}, p_{0}\right), \quad(\rho, u, v, p)_{2}=\left(2 \rho_{0}, u_{0}, v_{0}, p_{0}\right) \\
& (\rho, u, v, p)_{3}=\left(\rho_{0},-u_{0}, v_{0}, p_{0}\right), \quad(\rho, u, v, p)_{4}=\left(3 \rho_{0},-u_{0},-v_{0}, p_{0}\right)
\end{aligned}
$$

where $\rho_{0}=100 \mathrm{~kg} / \mathrm{m}^{3}, u_{0}=1000 \mathrm{~m} / \mathrm{s}, v_{0}=1000 \mathrm{~m} / \mathrm{s}$ and $p_{0}=40$ bars and the subscripts 1 to 4 indicate the quadrant in the two-dimensional plane where the initial value applies. The domain is the unit square and the simulations were carried out up to $200 \mu$ s using nitrogen as the gas, and discretized with $200 \times 200$ grid points.

Fig. 19 shows comparisons between the original and 'rs' extended Euler system; the latter using the formulation in $\S 7$. Results for the 'r' extended Euler system are not shown because they are indistinguishable from the original Euler system solutions. In this problem the dominant source of mechanical nonequilibrium is the presence of slip lines. We observe that the original Euler solutions develop strong density and pressure excursions along the four contacts lines in their respective quadrants. The peak pressure in the original Euler system (top row) is as high as $18 \mathrm{MPa}$ (180 bars), while the solution using the 'rs' extended Euler system 

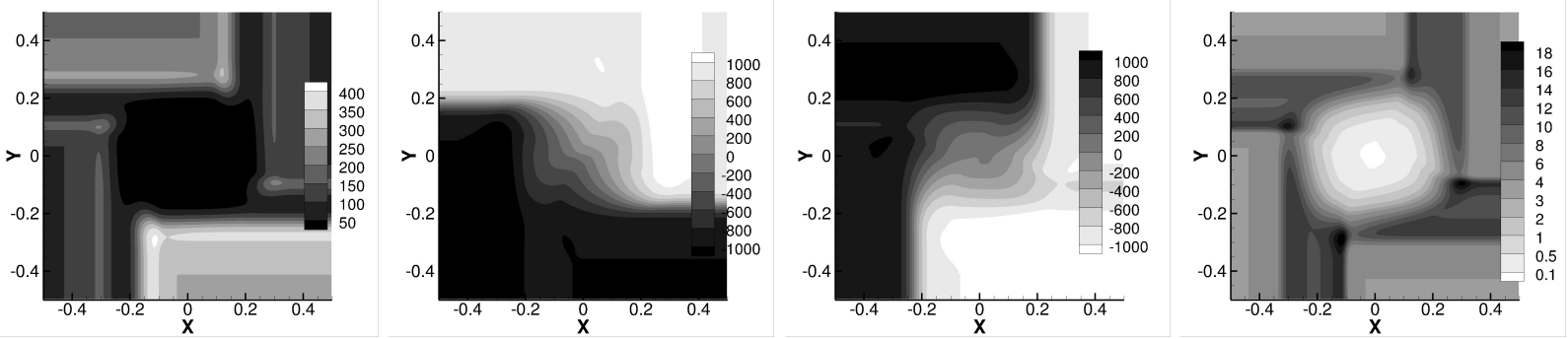

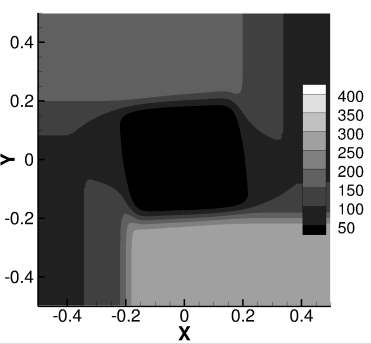

(a) $\rho-\mathrm{kg} / \mathrm{m}^{3}$

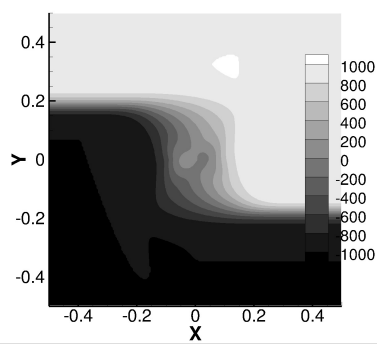

(b) $u-\mathrm{m} / \mathrm{s}$

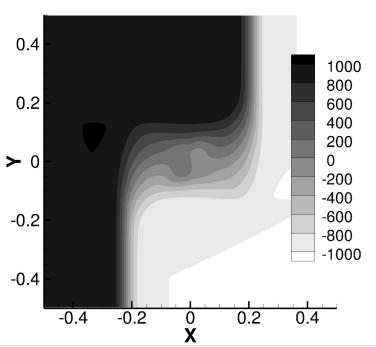

(c) $v-\mathrm{m} / \mathrm{s}$

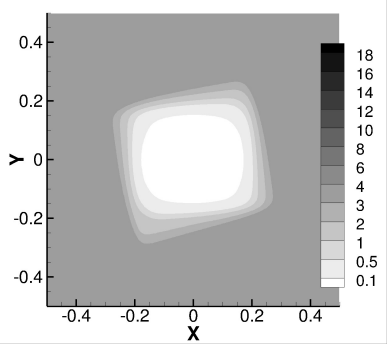

(d) $p-\mathrm{MPa}$

Figure 19: Two-dimensional Riemann problem at $t=200 \mu \mathrm{s}$. Original (top) and 'rs' extended Euler system (bottom)

(bottom row) preserves the peak pressure at $4 \mathrm{MPa}$ (40 bars). The contamination in the velocity is not as clear because the magnitude of velocity variation is confused with the multidimensional nature of the fields. Note also that at this pressure, near where the EOS is most nonlinear, we do not see the pattern shown in [7] with the slip lines twisting in a clock-wise manner. We have conducted simulations at higher pressure and obtain similar results to those of [7] but they are not reported here.

The present example highlights that our treatment of the transported kinetic energy equation, Eq. (78), is effective also in multiple dimensions.

\section{Conclusions}

Several new methods have been developed and studied to tackle the problem of violation of mechanical equilibrium at density interfaces of flows with real gas equations of state (van der Waals in the present case). The approach studied involves extra thermodynamic-like fields and their governing equations. The sole purpose of these fields is to relax the negative numerical effects of density nonlinearities in the EOS that are generally observed. The methods are fully conservative with respect to mass, momentum and total energy while it is nonconservative in the additional pressure-like fields. The approach is demonstrated for Roe 
and HLLC schemes of the Euler equations. At contact interfaces, the extra field has specific intermediate states that produce monotonic evolutions while at shocks the additional terms of its evolution equation induce variations that enable the recovery of the initial equation of state thermodynamics. The extended thermodynamics, and corresponding numerical treatment, enable contact discontinuities and strong shocks to be simulated accurately. Using existing mathematical framework, it is shown that the derived numerical methods can be expressed completely in analytical form as a function of the parameters of the real EOS. This has several advantages, including the ability to confirm the effect of the nonconservative term in the extra field equation and to study its convergence properties. Several demonstrations in one-dimensional contact and shock problems are presented and an application to jet injection in supercritical conditions in two dimensions is shown to produce excellent results.

An extension to treat negative numerical effects of strong slip lines using a transported kinetic energy equation in multiple dimensions has also been demonstrated. Again, the resulting equation for kinetic energy is non-conservative and its consistent treatment is described and simulations were carried out in one and two dimensional problems to highlight the benefits of this approach.

In the future we plan to consider more complex equations of state with nonlinear contributions related to both density and temperature, in the same frame of the conservative formulation.

\section{Acknowledgements}

The first author was supported in part by the Department of Energy, National Nuclear Security Administration, under award no. DE-NA0002382 and the California Institute of Technology. The authors are very grateful to Francois Fraysse (researcher at RS2N SAS) for his efficiency in the method coding in the unstructured code DALPHADT. Part of this work has been carried out in the framework of the Labex MEC (ANR-10-LABX-0092)and of the A*MIDEX project (ANR-11-IDEX-0001-02) funded by the Investissements

d'Avenir, French Government program managed by the French National Research Agency (ANR). We also acknowledge funding from ANR though project ANR-14-CE22-0014 (SUBSUPERJET). 


\section{Appendix A. van der Waal's equation of state}

We start from the van der Waal's equation of state, supposed to be accurate in the supercritical region

$$
p=\frac{N R^{o} T}{V-N \tilde{b}}-\tilde{a} \frac{N^{2}}{V^{2}}
$$

where we are using extensive properties of number of molecules $N$ and volume $V$ because in the gas-dynamics context, local equilibrium thermodynamics are applied to control volumes composed of material volumes where the number of fluid particles is constant. Therefore, $N$ is constant in the following derivations. We denote the extensive internal energy as $U(T, V)$ and write the general thermodynamic relationship

$$
d U=\tilde{C}_{V} d T+\left(\left.T \frac{\partial P}{\partial T}\right|_{V}-p\right) d V
$$

where

$$
\tilde{C}_{V}=\left.T \frac{\partial S}{\partial T}\right|_{V}
$$

Using Eq. (A.1) we have

$$
d U=\tilde{C}_{V} d T+\tilde{a} \frac{N^{2}}{V^{2}} d V
$$

and integrating we arrive at (assuming $\tilde{C}_{V}$ is only a function of $T$, which is reasonable for a gas, see below)

$$
U=\int_{T_{0}}^{T} \tilde{C}_{V} d T-\tilde{a} \frac{N^{2}}{V}+U_{0}
$$

This last assumption can be verified as follows. Using Maxwell second relation, we have

$$
\left.\frac{\partial P}{\partial T}\right|_{V}=\left.\frac{\partial S}{\partial V}\right|_{T}=\frac{N R^{o}}{V-N \tilde{b}}
$$

which can be integrated to give

$$
S=S_{0}(T)+N R^{o} \ln (V-N \tilde{b})
$$

so that Eq. (A.3) becomes

$$
\tilde{C}_{V}=\left.T \frac{\partial S}{\partial T}\right|_{V}=T \frac{d S_{0}}{d T}
$$

showing that $\tilde{C}_{V}$ is only a function of $T$ for a van der Waals gas. Then the entropy constant can be expressed as

$$
S_{0}(T)=S_{0}+\int_{T_{0}}^{T} \frac{\tilde{C}_{V}(T)}{T} d T
$$


where the $S_{0}$ in the right-hand side is a true constant and the complete entropy is given by

$$
S=\int_{T_{0}}^{T} \frac{\tilde{C}_{V}(T)}{T} d T+N R^{o} \ln (V-N \tilde{b})+S_{0}
$$

To complete the deductions, we require a caloric equation of state. This requires further knowledge or assumptions about $\tilde{C}_{V}$. If we assume that $\tilde{C}_{V}$ is constant independent of $T$ and introduce $\rho=W N / V$ where $W$ is the mass of a single molecule, then the equation of state becomes

$$
p=\frac{\rho R T}{1-\rho b}-a \rho^{2},
$$

where $R=R^{o} / W, b=\tilde{b} / W$ and $a=\tilde{a} / W^{2}$. The caloric equation of state is given by

$$
e=\frac{U}{N W}=C_{V} T-a \rho
$$

where the constant of integration have been ignored for simplicity and $C_{V}=\tilde{C}_{V} /(N W)$. Eliminating $T$ from Eq. (A.11) gives

$$
p=\frac{(\gamma-1)\left(\rho e+a \rho^{2}\right)}{1-\rho b}-a \rho^{2}
$$

where the apparent specific heat ratio $\gamma$ is defined as

$$
\gamma=\frac{\tilde{C}_{V}+N R^{o}}{\tilde{C}_{V}}=\frac{C_{V}+R}{C_{V}}
$$

which is different from the ratio of specific heats for a nonideal gas, as explained now. Using the definition of specific heat at constant pressure,

$$
\tilde{C}_{P}=\left.T \frac{\partial S}{\partial T}\right|_{P}=\tilde{C}_{V}+\left.\frac{N R^{o} T}{V-N \tilde{b}} \frac{\partial V}{\partial T}\right|_{P}=\tilde{C}_{V}+N R^{o}+\frac{N R^{o} \tilde{c}}{1-\tilde{c}}
$$

where

$$
\tilde{c}=2 \frac{\tilde{a} N(V-N \tilde{b})^{2}}{R^{o} T V^{3}}=2 \frac{a}{R T} \rho(1-b \rho)^{2} .
$$

Now, we can relate the apparent specific heat ratio to the true specific heat ratio $\tilde{\gamma}=\tilde{C}_{P} / \tilde{C}_{V}$ by

$$
\gamma=\frac{\tilde{C}_{P}-N R^{o} \tilde{c} /(1-\tilde{c})}{\tilde{C}_{V}}=\tilde{\gamma}-\frac{N R^{o} \tilde{c}}{\tilde{C}_{V}(1-\tilde{c})}=(1-\tilde{c}) \tilde{\gamma}+\tilde{c} .
$$

Finally, we proceed to define the speed of sound (for infinitesimal acoustic fluctuations) given by

$$
c_{\mathrm{th}}^{2}=\left.\frac{\partial p}{\partial 1 / V}\right|_{S}=-\left.\frac{V^{2}}{N} \frac{\partial p}{\partial V}\right|_{S}
$$


From the consideration that $p(V, S)$ and $p(V, T)$ we have

$$
d p=\left.\frac{\partial p}{\partial V}\right|_{S} d V+\left.\frac{\partial p}{\partial S}\right|_{V} d S=\left.\frac{\partial p}{\partial V}\right|_{T} d V+\left.\frac{\partial p}{\partial T}\right|_{V} d T .
$$

To eliminate the unknowns, we consider $S(T, V)$ and

$$
d S=\left.\frac{\partial S}{\partial T}\right|_{V} d T+\left.\frac{\partial S}{\partial V}\right|_{T} d V .
$$

Then we have

$$
\left.\frac{\partial p}{\partial V}\right|_{S}+\left.\left.\frac{\partial p}{\partial S}\right|_{V} \frac{\partial S}{\partial V}\right|_{T}=\left.\frac{\partial p}{\partial V}\right|_{T}
$$

and

$$
\left.\left.\frac{\partial p}{\partial S}\right|_{V} \frac{\partial S}{\partial T}\right|_{V}=\left.\frac{\partial p}{\partial T}\right|_{V} .
$$

From the already derives quantities, we can evaluate

$$
\left.\frac{\partial p}{\partial S}\right|_{V}=\frac{N R^{\circ} T}{(V-N \tilde{b}) \tilde{C}_{V}(T)},
$$

and then

$$
\left.\frac{\partial p}{\partial V}\right|_{S}=-\frac{N R^{o} T}{(V-N \tilde{b})^{2}} \frac{\tilde{C}_{V}+N R^{o}}{\tilde{C}_{V}}+2 \tilde{a} \frac{N^{2}}{V^{3}} .
$$

where $\tilde{\gamma}=\tilde{C}_{P} / \tilde{C}_{V}$, then we have for Eq. (A.24)

$$
\left.\frac{\partial p}{\partial V}\right|_{S}=-\gamma \frac{N R^{o} T}{(V-N \tilde{b})^{2}}+2 \tilde{a} \frac{N^{2}}{V^{3}} .
$$

Introducing this latest result in Eq. (A.18) gives

$$
c^{2}=\frac{\gamma R T}{(1-\rho b)^{2}}-2 a \rho=\frac{\gamma(\gamma-1)(e+\rho a)}{(1-\rho b)^{2}}-2 a \rho .
$$

It is also possible to use the traditional $\tilde{\gamma}$ to express the speed of sound, giving

$$
c^{2}=\tilde{\gamma}\left(\frac{R T}{(1-\rho b)^{2}}-2 a \rho\right)=\gamma\left(\frac{p / \rho+a \rho(2 \rho b-1)}{1-\rho b}\right) .
$$

\section{Appendix B. Generalized Roe averaged matrix}

To derive the matrix $\tilde{M}$ for the extended system, we start by calculating $J$, giving

$$
J=\left[\begin{array}{ccccc}
0 & 1 & 0 & 0 & 0 \\
p_{\rho}-u^{2} & 2 u+p_{\rho u} & p_{\rho v} & p_{\rho E} & p_{r} \\
-u v & v & u & 0 & 0 \\
u\left(p_{\rho}-H\right) & u p_{\rho u}+H & u p_{\rho v} & \left(1+p_{\rho E}\right) u & u p_{r} \\
-\left(\beta+\frac{r}{\rho}\right) u & \beta+\frac{r}{\rho} & 0 & 0 & u
\end{array}\right],
$$


where

$$
p_{Q_{i}}=\frac{\partial p}{\partial Q_{i}},
$$

and using Eq. (23) by replacing $\rho e=\rho E-\left[(\rho u)^{2}+(\rho v)^{2}\right] /(2 \rho)$, results in

$$
p=\Gamma(\rho)\left(\rho E-\frac{\left[(\rho u)^{2}+(\rho v)^{2}\right]}{2 \rho}-r\right),
$$

and gives

$$
p_{\rho}=\Gamma\left(\frac{u^{2}+v^{2}}{2}\right)+\frac{\Gamma^{\prime}}{\Gamma} p, \quad p_{\rho E}=\Gamma, \quad p_{\rho u}=-\Gamma u, \quad p_{\rho v}=-\Gamma v, \quad p_{r}=-\Gamma,
$$

where we have used Eq. (24). To proceed and calculate $M$ according to [24], we invert Eq. (41) to give

$$
Q_{o}(W)=\left\{w_{1}^{2}, w_{1} w_{2}, w_{1} w_{5}, w_{1} w_{3}-p(W), w_{1} w_{4}\right\}
$$

where $p$ can be written explicitly as a function of $w_{k}$ by

$$
p=Z\left[w_{1}\left(w_{3}-w_{4}\right)-\frac{1}{2}\left(w_{2}^{2}+w_{5}^{2}\right)\right],
$$

where $\Gamma=\Gamma\left(w_{1}^{2}\right)$ now and

$$
Z=\frac{\Gamma}{\Gamma+1}=\frac{\gamma-1}{\gamma-b \rho}
$$

Then,

$$
\frac{\partial Q_{o}}{\partial W}=\left[\begin{array}{ccccc}
2 w_{1} & 0 & 0 & 0 & 0 \\
w_{2} & w_{1} & 0 & 0 & 0 \\
w_{5} & 0 & w_{1} & 0 & 0 \\
w_{3}-p_{1} & -p_{2} & -p_{5} & w_{1}-p_{3} & -p_{4} \\
w_{4} & 0 & 0 & 0 & w_{1}
\end{array}\right]
$$

where

$$
p_{k}=\frac{\partial p}{\partial w_{k}}
$$

are given by

$$
\begin{aligned}
& p_{1}=Z\left\{\frac{2 b Z w_{1}}{\gamma-1}\left[w_{1}\left(w_{3}-w_{4}\right)-\frac{1}{2}\left(w_{2}^{2}+w_{5}^{2}\right)\right]+w_{3}-w_{4}\right\}, \\
& p_{2}=-Z w_{2}, \quad p_{5}=-Z w_{5}, \quad p_{3}=Z w_{1}, \quad p_{4}=-Z w_{1} .
\end{aligned}
$$


We can now calculate Eq. (38) in a straight-forward manner, giving

$$
\tilde{B}=\left[\begin{array}{ccccc}
2 \bar{w}_{1} & 0 & 0 & 0 & 0 \\
\bar{w}_{2} & \bar{w}_{1} & 0 & 0 & 0 \\
\bar{w}_{5} & 0 & \bar{w}_{1} & 0 & 0 \\
\bar{w}_{3}-\tilde{p}_{1} & -\tilde{p}_{2} & -\tilde{p}_{5} & \bar{w}_{1}-\tilde{p}_{3} & -\tilde{p}_{4} \\
\bar{w}_{4} & 0 & 0 & 0 & \bar{w}_{1}
\end{array}\right],
$$

where $\bar{w}_{k}$ denotes the average of the left and right states of $w_{k}$, and

$$
\tilde{p}_{k}=\int_{0}^{1} p_{k}\left(W_{L}+\left(W_{R}-W_{L}\right) s\right) d s .
$$

As observed by [24], the approximations for $\tilde{p}_{k}$ are not independent and they satisfy

$$
\Delta p=p_{r}-p_{l}=\sum \tilde{p}_{k} \Delta w_{k}
$$

which is very convenient to determine $\tilde{p}_{1}$, given the complexity of its formula (shown in Eq. (B.9)).

Next, we express the partial derivatives of $p$ with respect to $Q$ needed in Eq. (B.1). From the chain rule of differentiation we have

$$
\frac{\partial p}{\partial W}=\left(\frac{\partial Q_{o}}{\partial W}\right)^{T} \frac{\partial p}{\partial Q} \quad \rightarrow \quad \frac{\partial p}{\partial Q}=\left(\frac{\partial Q_{o}}{\partial W}\right)^{-T} \frac{\partial p}{\partial W}
$$

giving

$$
\begin{aligned}
p_{\rho} & =\frac{p_{1} w_{1}-p_{2} w_{2}-p_{5} w_{5}-p_{3} w_{3}-p_{4} w_{4}}{2 w_{1}\left(w_{1}-p_{3}\right)} \\
p_{\rho u} & =\frac{p_{2}}{w_{1}-p_{3}}, \quad p_{\rho v}=\frac{p_{5}}{w_{1}-p_{3}}, \quad p_{\rho E}=\frac{p_{3}}{w_{1}-p_{3}}, \quad p_{r}=\frac{p_{4}}{w_{1}-p_{3}} .
\end{aligned}
$$

Then, we have

$$
J \frac{\partial Q_{o}}{\partial W}=\left[\begin{array}{ccccc}
w_{2} & w_{1} & 0 & 0 & 0 \\
p_{1} & p_{2}+2 w_{2} & p_{5} & p_{3} & p_{4} \\
0 & w_{5} & w_{2} & 0 & 0 \\
0 & w_{3} & 0 & w_{2} & 0 \\
\kappa w_{2} w_{1}^{4}-\frac{w_{2} w_{4}}{w_{1}} & 2 w_{4}-\kappa w_{1}^{5} & 0 & 0 & w_{2}
\end{array}\right]
$$


which averages to

$$
\tilde{C}=\left[\begin{array}{ccccc}
\bar{w}_{2} & \bar{w}_{1} & 0 & 0 & 0 \\
\tilde{p}_{1} & \tilde{p}_{2}+2 \bar{w}_{2} & \tilde{p}_{5} & \tilde{p}_{3} & \tilde{p}_{4} \\
0 & \bar{w}_{5} & \bar{w}_{2} & 0 & 0 \\
0 & \bar{w}_{3} & 0 & \bar{w}_{2} & 0 \\
\left(\kappa \tilde{\alpha}_{2}-\tilde{\phi}\right) \bar{w}_{1} & \left(2 \hat{\omega}-\kappa \tilde{\alpha}_{1}\right) \bar{w}_{1} & 0 & 0 & \bar{w}_{2}
\end{array}\right]
$$

where $\hat{\omega}=\bar{w}_{4} / \bar{w}_{1}$ and

$$
\tilde{\alpha}_{1}=\frac{\overline{w_{1}^{5}}}{\bar{w}_{1}}, \quad \tilde{\alpha}_{2}=\frac{\overline{w_{2} w_{1}^{4}}}{\bar{w}_{1}}, \quad \tilde{\phi}=\frac{\overline{w_{2} w_{4} / w_{1}}}{\bar{w}_{1}}
$$

and generates the following generalized Roe-average matrix

$$
\tilde{M}=\tilde{C} \tilde{B}^{-1}=\left[\begin{array}{ccccc}
0 & 1 & 0 & 0 & 0 \\
\tilde{p}_{\rho}-\hat{u}^{2} & \tilde{p}_{\rho u}+2 \hat{u} & \tilde{p}_{\rho v} & \tilde{p}_{\rho E} & \tilde{p}_{r} \\
-\hat{u} \hat{v} & \hat{v} & \hat{u} & 0 & 0 \\
\left(\tilde{p}_{\rho}-\hat{H}\right) \hat{u} & \hat{H}+\tilde{p}_{\rho u} \hat{u} & \hat{u} \tilde{p}_{\rho v} & \left(1+\tilde{p}_{\rho E}\right) \hat{u} & \tilde{p}_{r} \hat{u} \\
\frac{1}{2}\left[\kappa \tilde{\alpha}_{2}-\tilde{\phi}+\hat{u}\left(\kappa \tilde{\alpha}_{1}-3 \hat{\omega}\right)\right] & 2 \hat{\omega}-\kappa \tilde{\alpha}_{1} & 0 & 0 & \hat{u}
\end{array}\right],
$$

where

$$
\begin{aligned}
\hat{u} & =\frac{\bar{w}_{2}}{\bar{w}_{1}}=\frac{\sqrt{\rho_{l}} u_{l}+\sqrt{\rho_{r}} u_{r}}{\sqrt{\rho_{l}}+\sqrt{\rho_{r}}}, \quad \hat{v}=\frac{\bar{w}_{5}}{\bar{w}_{1}}=\frac{\sqrt{\rho_{l}} v_{l}+\sqrt{\rho_{r}} v_{r}}{\sqrt{\rho_{l}}+\sqrt{\rho_{r}}}, \quad \hat{H}=\frac{\bar{w}_{3}}{\bar{w}_{1}}=\frac{\sqrt{\rho_{l}} H_{l}+\sqrt{\rho_{r}} H_{r}}{\sqrt{\rho_{l}}+\sqrt{\rho_{r}}}, \\
\hat{\omega} & =\frac{\bar{w}_{4}}{\bar{w}_{1}}, \quad \tilde{\alpha}_{1}=\frac{1}{3}\left(\rho_{L}-\sqrt{\rho_{L} \rho_{R}}+\rho_{R}\right)\left(\rho_{L}+\sqrt{\rho_{L} \rho_{R}}+\rho_{R}\right), \\
\tilde{\alpha}_{2} & =\frac{5\left(\rho_{L}^{5 / 2} u_{L}+\rho_{R}^{5 / 2} u_{R}\right)+\rho_{L}^{3 / 2} \rho_{R}\left(3 u_{L}+2 u_{R}\right)+\rho_{L}^{2} \sqrt{\rho_{R}}\left(4 u_{L}+u_{R}\right)}{15\left(\sqrt{\rho_{L}}+\sqrt{\rho_{R}}\right)} \\
& +\frac{\rho_{L} \rho_{R}^{3 / 2}\left(2 u_{L}+3 u_{R}\right)+\sqrt{\rho_{L}} \rho_{R}^{2}\left(u_{L}+4 u_{R}\right)}{15\left(\sqrt{\rho_{L}}+\sqrt{\rho_{R}}\right)}, \\
\tilde{\phi} & =\frac{\log \left(\rho_{R}\right)\left(u_{L}-u_{R}\right)\left(r_{R} \sqrt{\rho_{L}^{3} \rho_{R}}-r_{L} \sqrt{\rho_{L} \rho_{R}^{3}}\right)-\log \left(\rho_{L}\right)\left(u_{L}-u_{R}\right)\left(r_{R} \sqrt{\rho_{L}^{3} \rho_{R}}-r_{L} \sqrt{\rho_{L} \rho_{R}^{3}}\right)}{\left(\sqrt{\rho_{L}}-\sqrt{\rho_{R}}\right)^{3}\left(\sqrt{\rho_{L}}+\sqrt{\rho_{R}}\right) \sqrt{\rho_{L} \rho_{R}}} \\
& +\frac{r_{L} u_{L} \sqrt{\rho_{L}^{3} \rho_{R}}+3 r_{L} u_{L} \sqrt{\rho_{L} \rho_{R}^{3}}-4 \rho_{L} \rho_{R} r_{L} u_{L}+\rho_{L} \rho_{R} r_{L} u_{R}-\rho_{R}^{2} r_{L} u_{R}+\rho_{L}^{2} r_{R} u_{L}}{\left(\sqrt{\rho_{L}}-\sqrt{\rho_{R}}\right)^{3}\left(\sqrt{\rho_{L}}+\sqrt{\rho_{R}}\right) \sqrt{\rho_{L} \rho_{R}}} \\
& +\frac{4 \rho_{L} \rho_{R} r_{R} u_{R}-\rho_{L} \rho_{R} r_{R} u_{L}-3 r_{R} u_{R} \sqrt{\rho_{L}^{3} \rho_{R}}-r_{R} u_{R} \sqrt{\rho_{L} \rho_{R}^{3}}}{\left(\sqrt{\rho_{L}}-\sqrt{\rho_{R}}\right)^{3}\left(\sqrt{\rho_{L}}+\sqrt{\rho_{R}}\right) \sqrt{\rho_{L} \rho_{R}}}
\end{aligned}
$$


and the average partial derivatives $\tilde{p}_{Q}$ are defined by requiring that Eq. (B.14) applies to the averaged quantities, giving

$$
\begin{aligned}
\tilde{p}_{\rho} & =\frac{\tilde{p}_{1} \bar{w}_{1}-\tilde{p}_{2} \bar{w}_{2}-\tilde{p}_{5} \bar{w}_{5}-\tilde{p}_{3} \bar{w}_{3}-\tilde{p}_{4} \bar{w}_{4}}{2 \bar{w}_{1}\left(\bar{w}_{1}-\tilde{p}_{3}\right)} \\
\tilde{p}_{\rho u} & =\frac{\tilde{p}_{2}}{\bar{w}_{1}-\tilde{p}_{3}}, \quad \tilde{p}_{\rho v}=\frac{\tilde{p}_{5}}{\bar{w}_{1}-\tilde{p}_{3}}, \quad \tilde{p}_{\rho E}=\frac{\tilde{p}_{3}}{\bar{w}_{1}-\tilde{p}_{3}}, \quad \tilde{p}_{r}=\frac{\tilde{p}_{4}}{\bar{w}_{1}-\tilde{p}_{3}} .
\end{aligned}
$$

This simplification, not averaging Eq. (B.14) exactly, does not affect the accuracy of the resulting Roe matrix since it is easy to verify that we also have

$$
\Delta p=\tilde{p}_{\rho} \Delta \rho+\tilde{p}_{\rho u} \Delta \rho u+\tilde{p}_{\rho v} \Delta \rho v+\tilde{p}_{\rho E} \Delta \rho E+\tilde{p}_{r} \Delta r
$$

after using Eq. (B.12) and Eq. (37).

Note that the Euler submatrix of Eq. (B.18) is analogous to that of Eq. (31) if all quantities are replaced by the averaged values, as desired. Nonetheless, the addition of $r$ adds some complexity to the whole matrix and the coupling between fields as it is natural.

The complexity of the above formulas can be considerable for numerical implementation purposes. But, [24] noticed that for the conservation-form system of the Euler equations, further simplifications are possible since the Roe matrix is only required to satisfy the three equations in Eq. (6), while the matrix $M$ has nine elements. Multiple degrees of freedom are available but not all produce hyperbolic systems. Considering these flux jump conditions, and the form of Eq. (B.18), one can see that as long as Eq. (B.25) is ensured, the flux jumps $\Delta(\rho u)$ and $\Delta(H u)$ are unmodified.

We now extend the procedure in [24] to the quasi-conservative system and define the approximate pressure derivatives with respect to $W$ by

$$
\tilde{p}_{1}=(\hat{H}-\hat{\omega}+2 \hat{\alpha}) \tilde{p}_{3}, \quad \tilde{p}_{2}=-\hat{u} \tilde{p}_{3}, \quad \tilde{p}_{5}=-\hat{v} \tilde{p}_{3}, \quad \tilde{p}_{3}=\tilde{Z} \bar{w}_{1}, \quad \tilde{p}_{4}=-\tilde{p}_{3},
$$

where

$$
\tilde{\Gamma}=\int_{0}^{1} \Gamma(W(s)) d s
$$

and

$$
\tilde{Z}=\frac{\tilde{\Gamma}}{\tilde{\Gamma}+1}
$$


and $\hat{\alpha}$ is a free parameter determined by enforcing Eq. (B.12) and using Eq. (37), resulting in

$$
\begin{aligned}
\Delta p & =\tilde{p}_{3}\left[(\hat{H}-\hat{\omega}+2 \hat{\alpha}) \Delta w_{1}-\hat{u} \Delta w_{2}-\hat{v} \Delta w_{5}+\Delta w_{3}-\Delta w_{4}\right] \\
& =\tilde{Z}\left[\left(\bar{w}_{3}-\bar{w}_{4}+2 \bar{w}_{1} \hat{\alpha}\right) \Delta w_{1}-\bar{w}_{2} \Delta w_{2}-\bar{w}_{5} \Delta w_{5}+\bar{w}_{1} \Delta w_{3}-\bar{w}_{1} \Delta w_{4}\right] \\
& =\tilde{\Gamma}\left[\left(\hat{\alpha}+\frac{1}{2}\left(\hat{u}^{2}+\hat{v}^{2}\right)\right) \Delta \rho-\hat{u} \Delta \rho u-\hat{v} \Delta \rho v+\Delta \rho E-\Delta r\right],
\end{aligned}
$$

which can be simplified by using the identity

$$
\frac{1}{2} \Delta\left(\rho u^{2}\right)=\hat{u} \Delta \rho u-\frac{1}{2} \hat{u}^{2} \Delta \rho
$$

and $\Delta(\rho h)=\Delta(\rho e)+\Delta p$, to

$$
\Delta p=\tilde{\Gamma}(\Delta \rho h-\Delta p+\hat{\alpha} \Delta \rho-\Delta r),
$$

which can be used to eliminate $\hat{\alpha}$, giving

$$
\hat{\alpha}=\frac{1}{\tilde{Z}} \frac{\Delta p}{\Delta \rho}-\frac{\Delta \rho h}{\Delta \rho}+\frac{\Delta r}{\Delta \rho} .
$$

Eq. (B.33) is applicable as long s $\Delta \rho$ is not zero. Here, we deviate from [24], since the limit $\Delta \rho \rightarrow 0$ does not makes sense in Eq. (B.33) because the terms involved are not pure thermodynamic quantities. When this happens, we must revert to the calculation of $\tilde{p}_{k}$ according to Eq. (B.11) using Eq. (B.9), with the condition that $\Delta w_{1}=0$. The averaged $\tilde{p}_{k}$ for $k=2$ through 4 in Eq. (B.26) become exact and $\tilde{p}_{1}$ is given by

$$
\tilde{p}_{1}=Z\left[\frac{2 Z b w_{1}}{\gamma-1}\left(w_{1}\left(\bar{w}_{3}-\bar{w}_{4}\right)-\frac{1}{2}\left(\overline{w_{2}^{2}}+\overline{w_{5}^{2}}\right)\right)+\bar{w}_{3}-\bar{w}_{4}\right]
$$

where we have removed the tilde and overbar over $\Gamma$ and $w_{1}$, respectively, to emphasize that these averaged and pointwise values are identical because they depend only on $\rho$ which is constant when $\Delta \rho=0$. Now, Eq. (B.34) and the first of Eq. (B.26) give

$$
\hat{\alpha}=\frac{1}{2}\left(\frac{\tilde{p}_{1}}{\tilde{p}_{3}}-\hat{H}+\hat{\omega}\right) .
$$

Finally, introducing all the previous expressions in Eq. (B.18), gives the final matrix

$$
\tilde{M}=\left[\begin{array}{ccccc}
0 & 1 & 0 & 0 & 0 \\
\frac{\Gamma}{2}\left(\hat{u}^{2}+\hat{v}^{2}+2 \hat{\alpha}\right)-\hat{u}^{2} & (2-\tilde{\Gamma}) \hat{u} & -\hat{v} \tilde{\Gamma} & \tilde{\Gamma} & -\tilde{\Gamma} \\
-\hat{u} \hat{v} & \hat{v} & \hat{u} & 0 & 0 \\
\left(\tilde{\Gamma}\left(\hat{\alpha}+\frac{\hat{u}^{2}+\hat{v}^{2}}{2}\right)-\hat{H}\right) \hat{u} & \hat{H}-\tilde{\Gamma} \hat{u}^{2} & -\hat{u} \hat{v} \tilde{\Gamma} & (\tilde{\Gamma}+1) \hat{u} & -\tilde{\Gamma} \hat{u} \\
\frac{1}{2}\left[\kappa \tilde{\alpha}_{2}-\tilde{\phi}+\hat{u}\left(\kappa \tilde{\alpha}_{1}-3 \hat{\omega}\right)\right] & 2 \hat{\omega}-\kappa \tilde{\alpha}_{1} & 0 & 0 & \hat{u}
\end{array}\right],
$$


which has sound speed (squared) for the contact situation given by

$$
c_{o}^{2}=\tilde{\Gamma}\left[\hat{H}-2 \hat{\omega}-\frac{\left(\hat{u}^{2}+\hat{v}^{2}\right)}{2}+\hat{\alpha}+\kappa \tilde{\alpha}_{1}\right] .
$$

[1] E. Lee, H. Hornig, J. Kury, Adiabatic expansion of high explosive detonation products., Tech. rep., California Univ., Livermore. Lawrence Radiation Lab. (1968).

[2] T. Schmitt, L. Selle, A. Ruiz, B. Cuenot, Large-eddy simulation of supercritical-pressure round jets, AIAA Journal 48 (9) (2010) 2133-2144.

[3] H. Terashima, S. Kawai, N. Yamanishi, High-resolution numerical method for supercritical flows with large density variations, AIAA Journal 49 (12) (2011) 2658-2672.

[4] H. Terashima, M. Koshi, Approach for simulating gas-liquid-like flows under supercritical pressures using a high-order central differencing scheme, J. Comput. Phys. 231 (20) (2012) 6907-6923.

[5] H. Terashima, S. Kawai, M. Koshi, Consistent numerical diffusion terms for simulating compressible multicomponent flows, Computers and Fluids 88 (2013) 484-495.

[6] R. Abgrall, How to prevent pressure oscillations in multicomponent flow calculations: A quasi conservative approach, J. Comput. Phys. 125 (1) (1996) 150-160.

[7] K.-M. Shyue, A fluid-mixture type algorithm for compressible multicomponent flow with van der Waals equation of state, Journal of Computational Physics 156 (1) (1999) 43-88.

[8] R. Saurel, E. Franquet, E. Daniel, O. Le Metayer, A relaxation-projection method for compressible flows. part i: The numerical equation of state for the euler equations, Journal of Computational Physics 223 (2) (2007) 822-845.

[9] T. Gallouët, J.-M. Hérard, N. Seguin, A hybrid scheme to compute contact discontinuities in onedimensional Euler systems, Mathematical Modelling and Numerical Analysis 36 (6) (2002) 1133-1159.

[10] B. Lee, E. Toro, C. Castro, N. Nikiforakis, Adaptive Osher-type scheme for the Euler equations with highly nonlinear equations of state, J. Comput. Phys. 246 (1) (2013) 165-183. 
[11] S. Karni, Multicomponent flow calculations by a consistent primitive algorithm, Journal of Computational Physics 112 (1) (1994) 31-43.

[12] E. Toro, C. Castro, B. Lee, A novel numerical flux for the 3D Euler equations with general equation of state, Journal of Computational Physics 303 (2015) 80-94.

[13] K.-M. Shyue, An efficient shock-capturing algorithm for compressible multicomponent problems, J. Comput. Phys. 142 (1) (1998) 208-242.

[14] R. Saurel, R. Abgrall, A simple method for compressible multifluid flows, SIAM J. Sci. Comput. 21 (3) (1999) 1115-1145.

[15] K.-M. Shyue, A fluid-mixture type algorithm for compressible multicomponent flow with mie-grüneisen equation of state, Journal of Computational Physics 171 (2) (2001) 678-707.

[16] R. Saurel, R. Abgrall, A multiphase Godunov method for compressible multifluid and multiphase flows, Journal of Computational Physics 150 (2) (1999) 425-467.

[17] A. Kapila, R. Menikoff, J. Bdzil, S. Son, D. Stewart, Two-phase modeling of deflagration-to-detonation transition in granular materials: Reduced equations, Physics of Fluids 13 (10) (2001) 3002-3024.

[18] G. Allaire, S. Clerc, S. Kokh, A five-equation model for the simulation of interfaces between compressible fluids, Journal of Computational Physics 181 (2) (2002) 577-616.

[19] J. Massoni, R. Saurel, B. Nkonga, R. Abgrall, Proposition de méthodes et modèles eulériens pour les problèmes à interfaces entre fluides compressibles en présence de transfert de chaleur: Some models and eulerian methods for interface problems between compressible fluids with heat transfer, International Journal of Heat and Mass Transfer 45 (6) (2002) 1287-1307.

[20] A. Murrone, H. Guillard, A five equation reduced model for compressible two phase flow problems, Journal of Computational Physics 202 (2) (2005) 664-698.

[21] R. Saurel, F. Petitpas, R. Berry, Simple and efficient relaxation methods for interfaces separating compressible fluids, cavitating flows and shocks in multiphase mixtures, Journal of Computational Physics 228 (5) (2009) 1678-1712. 
[22] A. Harten, P. Lax, B. van Leer, On upstream differencing and Godunov-type schemes for hyperbolic conservation laws, SIAM Review 25 (1) (1983) 35-61.

[23] P. Roe, Approximate Riemann solvers, parameter vectors, and difference schemes, J. Comput. Phys. 43 (2) (1981) 357-372.

[24] I. Toumi, A weak formulation for Roe's approximate Riemann solver, Journal of Computational Physics 102 (2) (1992) 360-373.

[25] D. Ambrose, C. Tsonopoulos, Vapor-liquid critical properties of elements and compounds. 2. Normal Alekenes, J. Chem. Eng. Data 40 (1995) 531-546.

[26] N. I. of Standards, Technology, Webbook.

[27] T. Schmitt, private communication, test problem recommendation (2015).

[28] A. Harten, B. Engquist, S. Osher, S. Chakravarthy, Uniformly high order accurate essentially nonoscillatory scheems, III, Journal of Computational Physics 71 (2) (1987) 231-303.

[29] C.-W. Shu, S. Osher, Efficient implementation of essentially non-oscillatory shock-capturing schemes, Journal of Computational Physics 77 (2) (1988) 439-471.

[30] R. Loubere, M. Dumbser, S. Diot, A new family of high order unstructured MOOD and ADER finite volume schemes for multidimensional sys-tems of hyperbolic conservation laws, Communications in Computational Physics 16 (3) (2014) 718-763.

[31] R. K. Shukla, C. Pantano, J. B. Freund, An interface capturing method for the simulation of multi-phase compressible flows, Journal of Computational Physics 229 (19) (2010) 7411 - 7439. doi:10.1016/j.jcp.2010.06.025.

URL http://www.sciencedirect.com/science/article/pii/S0021999110003402

[32] A. Tiwari, J. B. Freund, C. Pantano, A diffuse interface model with immiscibility preservation, J. Comput. Phys. 252 (2013) 290-309.

[33] K.-M. Shyue, F. Xiao, An Eulerian interface sharpening algorithm for compressible two-phase flow: the algebraic THINC approach, Journal of Computational Physics 268 (2014) 326-354. 
[34] P. Lax, B. Wendroff, Systems of conservation laws, Communications on pure and applied mathematics 13 (2) (1960) 217-237.

[35] A. Guardone, L. Vigevano, Roe linearization for the van der waals gas, Journal of Computational Physics 175 (1) (2002) 50-78.

[36] P. Cinnella, Roe-type schemes for dense gas flow computations, Computers \& fluids 35 (10) (2006) $1264-1281$.

[37] P. Lax, Hyperbolic systems of conservation laws, SIAM, 1987.

[38] C. Dafermos, Hyperbolic conservation laws in continuum physics, Springer, 2005.

[39] B. Hayes, P. LeFloch, Nonclassical shocks and kinetic relations: strictly hyperbolic systems, SIAM J. Math. Anal. 31 (5) (2000) 941-991.

[40] G. DalMaso, P. LeFloch, F. Murat, Definition and weak stability of nonconservative products, Journal de Mathematiques pures et appliquees 74 (6) (1995) 483-548.

[41] A. Volpert, The space BV and quasilinear equations, Math. USSR 2 (1967) 257-267.

[42] R. Abgrall, S. Karni, A comment on the computation of non-conservative products, J. Comput. Phys. 229 (8) (2010) 2759-2763.

[43] E. Toro, M. Spruce, W. Spears, Restoration of the contact surface in the HLL-Riemann solver, Shock Waves 4 (1) (1994) 25-34.

[44] R. Saurel, A. Chinnayya, F. Renaud, Thermodynamic analysis and numerical resolution of a turbulent - fully ionized plasma flow model, Shock Waves 13 (4) (2003) 283-297.

[45] M. Castro, P. LeFloch, M. Muñoz-Ruiz, C. Parés, Why many theories of shock waves are necessary: Convergence error in formally path-consistent schemes, Journal of Computational Physics 227 (17) (2008) 8107-8129.

[46] R. Abgrall, B. Nkonga, R. Saurel, Efficient numerical approximation of compressible multi-material flow for unstructured meshes, Computers \& Fluids 32 (4) (2003) 571-605. 